\title{
Enhancement of the attitude dynamics capabilities of the spinning spacecraft using inertial morphing
}

\author{
P.M. Trivailo \\ pavel.trivailo@rmit.edu.au \\ School of Engineering \\ RMIT University \\ GPO Box 2476V, Melbourne, Victoria, 3001 \\ Australia \\ H. Kojima \\ hkojima@tmu.ac.jp \\ Department of Aeronautics and Astronautics \\ Tokyo Metropolitan University \\ Asahigaoka 6-6, Hino, Tokyo \\ Japan
}

\section{ABSTRACT}

In the previous works by the authors, an efficient method of control of the inversion of the spinning spacecraft was proposed. This method was prompted by the Dzhanibekov's Effect or Tennis Racket Theorem, which are often seen by many as odd or even mysterious. For the spacecraft, initially undergoing periodic flipping motion, proposed method allows to completely stop these flips by transferring the unstable motion into the regular stable spin. Similarly, the method allows activation of the flipping motion of the spacecraft, which is initially undergoing its stable spin. In this paper, spacecraft designs, which have inertial morphing capabilities, are considered and their advantages are further investigated. For general formulation, the ability of the spacecraft to change its inertial properties, associated with all three principal axes of inertia, are assumed. For simulation of these types of spacecraft systems, extended Euler's equations are used and peculiar dynamics of the spacecraft is illustrated with a several study cases. Complex spacecraft attitude dynamics manoeuvres, using geometric interpretation, employing angular momentum spheres and kinetic energy ellipsoids, are considered in detail. Contributions of the inertial morphing to the changes of the shape of the kinetic energy ellipsoid are demonstrated and are related to the resultant various feature manoeuvres, including inversion and re-orientation. A method of reduction of the compound rotation of the spacecraft into a single stable predominant rotation around one 
of the body axes was proposed. This is achieved via multi-stage morphing and employing proposed instalment into separatrices. Implementation of the morphing control capabilities are discussed. For the periodic inversion motions, calculation of the periods of the flipping motion, based on the complete elliptic integral of the first kind, is performed. Flipping periods for various combinations of inertial properties of the spacecraft are presented in a systematic way. This paper discusses strategies to the increase or reduction the flipping and/or wobbling motions. A discovered asymmetric ridge of high periods for peculiar combinations of the inertial properties is discussed in detail. Numerous examples are illustrated with animations in virtual reality, facilitating explanation of the analysis and control methodologies to a wide audience, including specialists, industry and students.

Keywords: Spacecraft attitude dynamics; Euler's equations of motion; morphing spacecraft; reaction wheel (RW); angular momentum sphere; kinetic energy ellipsoid; flipping period

\subsection{INTRODUCTION}

Prompted by an interesting phenomenon, known as the Dzhanibekov's Effect and Tennis Racquet Theorem, this paper, along with previous works ${ }^{(1-5)}$, aims to contribute to the attitude dynamics and control of spacecraft. Developing numerical simulation tools and analysis to explore these phenomenon and its geometric interpretation, it was possible to discover a new concept of the inertial morphing of the spacecraft systems to effectively control the Dzhanibekov's Effect ${ }^{(6)}$, presented in the recent works ${ }^{(1-5)}$ by the authors and extend it further to enable attitude control of the spinning/tumbling systems, converting compound motions into simple spins about one of the selected/nominated body axes.

\subsection{EULER'S DYNAMIC EQUATIONS FOR THE MOTION OF A RIGID BODY}

The spacecraft systems with constant values of the principal moments of inertia are considered first. For their analysis, the Euler dynamic equations of motion of the rigid body are used $^{(7)}$ :

$$
\left\{\begin{array}{l}
I_{x x} \dot{\omega}_{x}=\left(I_{y y}-I_{z z}\right) \omega_{y} \omega_{z} \\
I_{y y} \dot{\omega}_{y}=\left(I_{z z}-I_{x x}\right) \omega_{z} \omega_{x} \\
I_{z z} \dot{\omega}_{z}=\left(I_{x x}-I_{y y}\right) \omega_{x} \omega_{y}
\end{array}\right.
$$

These differential equations can be easily solved numerically providing that particular initial conditions are specified. One of the possible techniques is based on the Runge-Kutta methods, implemented in MATLAB and Cleve Moler, founder of Mathworks ${ }^{\circledR}$, provides interactive tools to implement this strategy ${ }^{(8)}$. Another possibility is to re-write Eqs. (1) in terms of the quaternions and solve these resultant equations.

\subsection{NON-DIMENSIONAL FORMULATION OF THE EQUATIONS}

For the main derivations in this paper it will be typically assumed that the system has three distinct principal moments of inertia, which are arranged in the following order: $I_{x x}<I_{y y}<I_{z z}$. 
For more generic formulations, two non-dimensional parameters, $\eta$ and $\xi$, both restricted in their values within the range between 0 and 1 are introduced:

$$
\eta=\frac{I_{x x}}{I_{z z}} ; \quad \xi=\frac{I_{y y}-I_{x x}}{I_{z z}-I_{x x}} ; \quad(0<\eta<1 ; \quad 0<\xi<1)
$$

Parameter $\xi$ in this case would have a similar meaning of the non-dimensional coordinate counterpart from the Finite Element Method, defining the current position within the finite element. In the context of this study, $\xi$ is specifying the non-dimensional relative position coordinate of the intermediate value of the moment of inertia between the minimum value of the moment of inertia $I_{x x}$ and the maximum value of the moment of inertia $I_{z z}$. In other words, it can be said that $\xi$ is the non-dimensional parameter in the Hermite functions, enabling calculation of $I_{y y}$ using $I_{x x}$ and $I_{z z}$, using the following relationship:

$$
I_{y y}=I_{x x}(1-\xi)+I_{z z} \xi
$$

The zero value of $\xi$ would now correspond to $I_{x x}$ and unit value of $\xi$ would correspond to $I_{z z}$

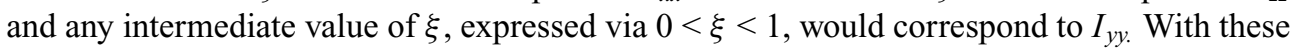
notations, we can also derive several relationships, enabling useful conversions in the future:

$$
I_{y y}=I_{x x}\left(1-\xi+\frac{1}{\eta}\right) ; \quad I_{z z}=\frac{I_{x x}}{\eta} .
$$

For illustration purposes, we solve Euler's Eqs. (1) for the same system with $I_{x x}=2 ; I_{y y}=$ $3 ; I_{z z}=4$ [all in $\mathrm{kg} \times \mathrm{m}^{2}$ ], which corresponds to $\xi=0.5$ and $\eta=0.5$, but consider three contrast cases of the initial conditions. An example of the results of the numerical simulations for $\omega_{x}, \omega_{y}, \omega_{z}$ are shown in Fig. 1.

For the more general interpretation, the introduction of non-dimensional angular momentum coordinates is proposed:

$$
\begin{aligned}
& \bar{H}_{x}(t)=H_{x} / H_{0}=I_{x x} \omega_{x} / \sqrt{\left(I_{x x} \omega_{x}\right)^{2}+\left(I_{y y} \omega_{y}\right)^{2}+\left(I_{z z} \omega_{z}\right)^{2}} \\
& \bar{H}_{y}(t)=H_{y} / H_{0}=I_{y y} \omega_{y} / \sqrt{\left(I_{x x} \omega_{x}\right)^{2}+\left(I_{y y} \omega_{y}\right)^{2}+\left(I_{z z} \omega_{z}\right)^{2}} \\
& \bar{H}_{z}(t)=H_{z} / H_{0}=I_{z z} \omega_{z} / \sqrt{\left(I_{x x} \omega_{x}\right)^{2}+\left(I_{y y} \omega_{y}\right)^{2}+\left(I_{z z} \omega_{z}\right)^{2}}
\end{aligned}
$$

where $H_{0}$ is the initial value of the angular momentum of the system, and $H_{x}, H_{y}$, and $H_{z}$ are its components in the body axes system $x, y$ and $z$. Non-dimensional coordinates $\bar{H}_{x}, \bar{H}_{y}, \bar{H}_{z}$ enable us to express the law of conservation of the angular momentum in the compact form

$$
\bar{H}_{x}^{2}+\bar{H}_{y}^{2}+\bar{H}_{z}^{2}=1
$$

Equation (6) can be conveniently interpreted as a unit sphere, known as the angular momentum sphere, AMS. This concept will be consistently used in this paper to illustrate simulation results. For example, Fig. 2 provides with an alternative interpretation of the previous cases $A$, $B$ and $C$ with polhodes ${ }^{(7)}-$ trajectories of the tips of the non-dimensional angular momentum vectors, $\overline{\mathbf{H}}_{A}, \overline{\mathbf{H}}_{B}$ and $\overline{\mathbf{H}}_{C}$ (these three polhodes are marked with the red color), residing on the AMS. Figure 2 also shows three superimposed quiver plots for these $\overline{\mathbf{H}}_{A}, \overline{\mathbf{H}}_{B}$ and $\overline{\mathbf{H}}_{C}$ vectors. 


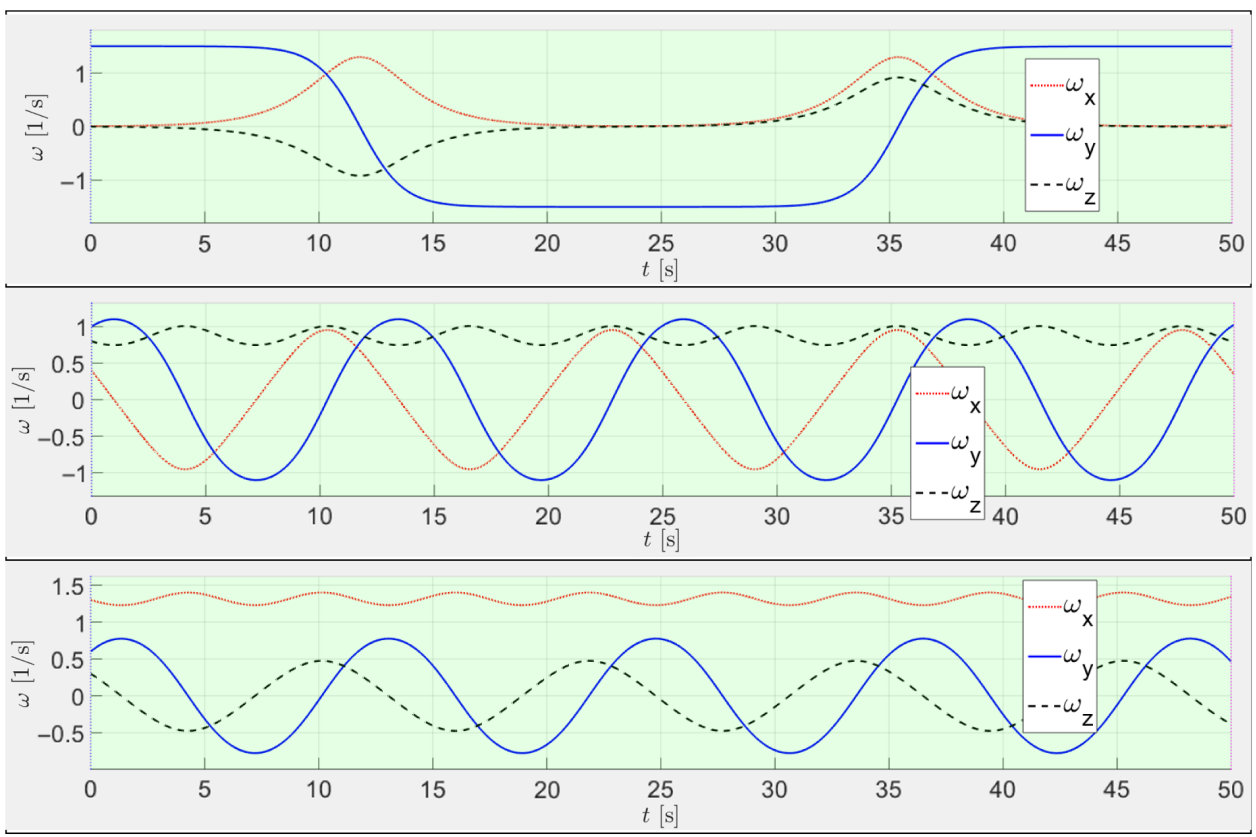

Figure 1. Time histories for angular velocity components $\omega_{x}, \omega_{y}, \omega_{z}$ for three contrast cases of initial conditions: $(\mathbf{A}) \omega_{x 0}=0.01, \omega_{y 0}=1.5, \omega_{z 0}=0.01 ;(\mathbf{B}) \omega_{x 0}=0.4, \omega_{y 0}=1, \omega_{z 0}=0.8 ;(\mathbf{C}) \omega_{x 0}=1.3, \omega_{y 0}=0.6, \omega_{z 0}=0.3$ (here and further all angular velocities are given in $\mathrm{rad} / \mathrm{s}$ ).

(a)

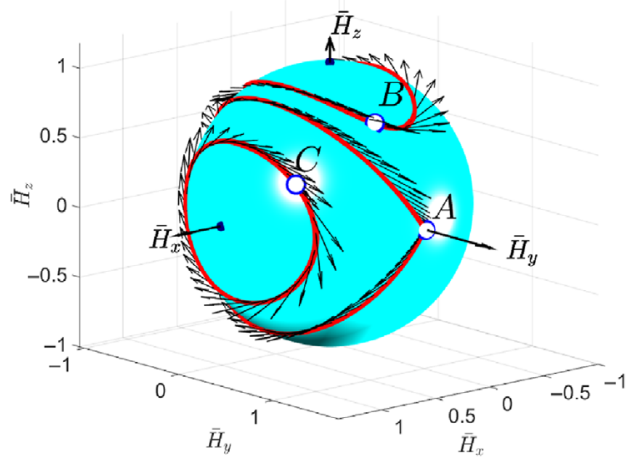

(b)

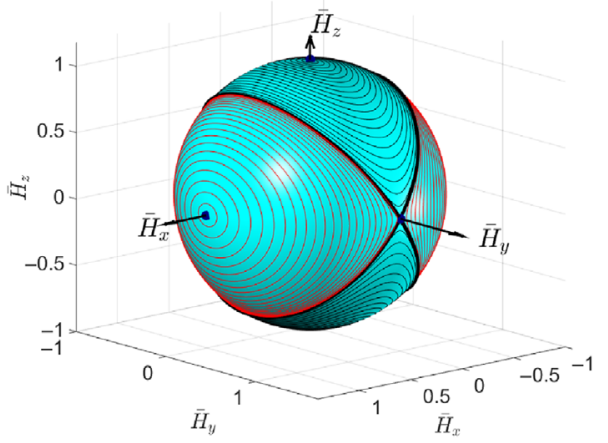

Figure 2. Polhodes: (a) for demonstration cases $A, B$ and $C$ in Fig. 1; (b) examples of broad coverage of initial conditions.

\subsection{POLHODES AND SEPARATRICES}

Figure $2 \mathrm{~b}$ shows that the polhodes can be split into four groups, separated by the (shown as bold black) lines, called separatrices. In case of $I_{y y}$ being the intermediate moment of inertia, two separatrices intersect at the points on the $y$ axis. It is possible to show that polhodes are seen on orthogonal projections as ellipses, hyperbolas and ellipses, and separatrices are seen on the $x-z$ projection as two lines, passing through the $y$ axis, reduced on projection to a dot 

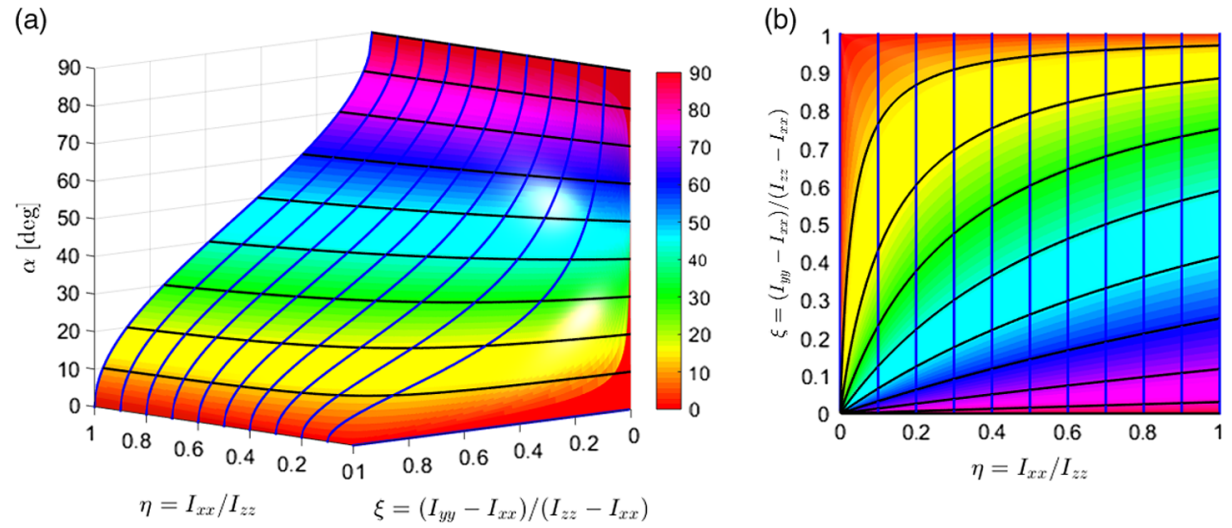

Figure 3. Changes in the angle $\alpha$ due to the variation in both, $\eta$ and $\xi$ : (a) 3D surface plot for $\alpha(\eta, \xi)$ function with colorbar added; (b) 2D projection of the $\alpha(\eta, \xi)$ surface with its contour lines: $\eta=0: 0.1: 1$; $\alpha=0: 10: 90$.

point. We can calculate an important characteristic of the rotational motion of the spacecraft, $\alpha$ - angle of inclination of the separatrix plane with respect to the $z \operatorname{axis}^{(3)}$ :

$$
\alpha=\arctan \left(\sqrt{\frac{I_{x}\left(I_{y}-I_{z}\right)}{I_{z}\left(I_{x}-I_{y}\right)}}\right)=\arctan \left(\sqrt{\eta\left(\frac{1}{\xi}-1\right)}\right)
$$

(only applicable for the $I_{x x}<I_{y y}<I_{z z}$ notations and $0<\eta<1$ and $0<\xi<1$ )

Changes in the angle $\alpha$ due to the variation in both, $\eta$ and $\xi$, are shown in Fig. 3. Note, that for convenience, values of $\alpha$ angles are presented in degrees.

The method, described in ${ }^{(3)}$, was based on the calculation of the value of the intermediate moment of inertia $I_{y y}$ for the specified angle $\alpha$ and known values of $I_{x x}=2.4$ and $I_{z z}=3.15$. For this formulation, Eq. (7) can be re-written as follows:

$$
\xi=\left\{1+\left[(\tan \alpha)^{2} / \eta\right]\right\}^{-1}
$$

In the particular case considered in reference ${ }^{(4)}$, for $I_{x x}=2.4$ and $I_{z z}=3.15$, the corresponding value of $\eta$ is equal to $\eta=0.7619$; furthermore, Eq. (8) gives $\xi=0.5907$, which (as per Eq. (3)), corresponds to $I_{x x}=2.8430$.

The generic graphical method, corresponding to this procedure, is illustrated in Fig. 4, where angle $\alpha$ (shown in degrees) is plotted as a function of $\xi$ for various values of $\eta=[.1$, $.2, .3, .4, .5, .6, .7, .8, .9,1]$.

\subsection{KINETIC ENERGY ELLIPSOID AND GEOMETRIC INTERPRETATION OF POLHODES}

The kinetic energy of the rotating body can be expressed in terms of the angular momentum components, such as follows:

$$
\frac{1}{2} I_{x x} \omega_{x}^{2}+\frac{1}{2} I_{y y} \omega_{y}^{2}+\frac{1}{2} I_{z z} \omega_{z}^{2}=\left[\frac{H_{x}(t)}{\sqrt{2 I_{x x}}}\right]^{2}+\left[\frac{H_{y}(t)}{\sqrt{2 I_{y y}}}\right]^{2}+\left[\frac{H_{z}(t)}{\sqrt{2 I_{z z}}}\right]^{2}=K(t)
$$




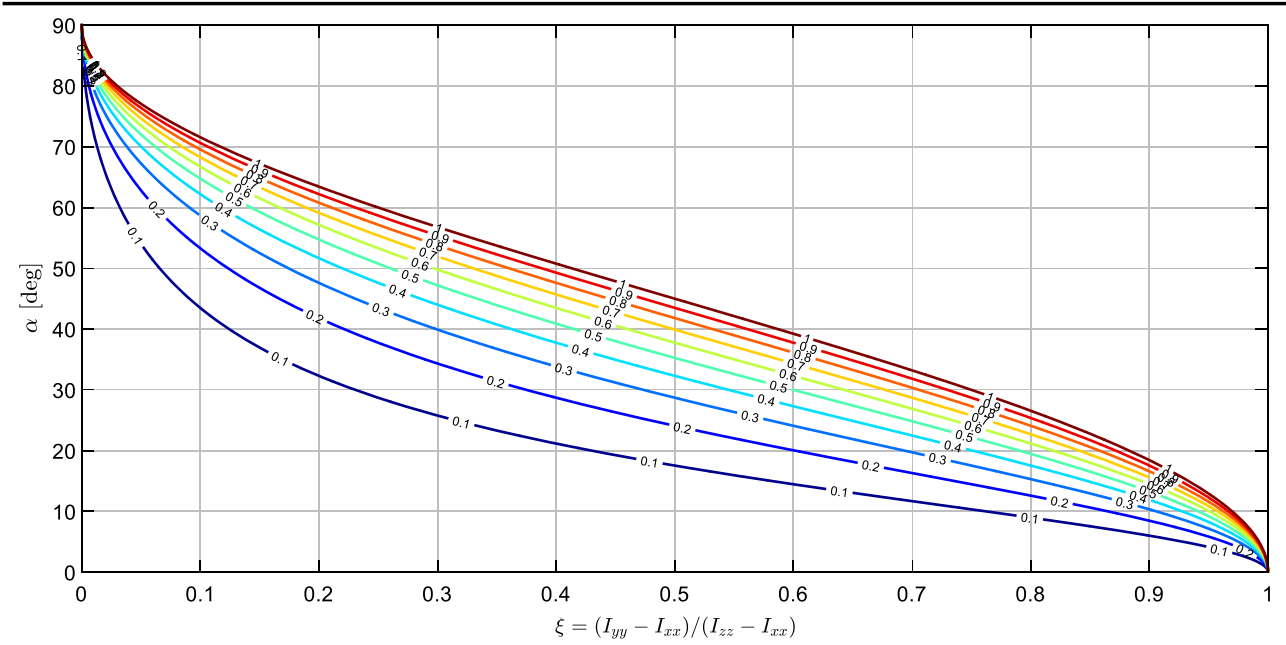

Figure 4. Changes in the angle $\alpha$ due to the variation in $\xi$ for selected values of $\eta=[1: 10] / 10$.

In the context of the inertial morphing concept (presented later in the paper), it's essential to consider the general case allowing for the principal moments of inertia of the system to change with time:

$$
\left[\frac{H_{x}(t)}{\sqrt{2 I_{x x}(t)}}\right]^{2}+\left[\frac{H_{y}(t)}{\sqrt{2 I_{y y}(t)}}\right]^{2}+\left[\frac{H_{z}(t)}{\sqrt{2 I_{z z}(t)}}\right]^{2}=K(t)
$$

Being dedicated to the non-dimensional formulation, we divide both sides of this equation by the constant $H_{0}{ }^{2}$ and rearrange result in terms of non-dimensional quantities $\bar{H}_{x}, \bar{H}_{y}$ and $\bar{H}_{z}$ :

$$
\left[\frac{H_{x}(t)}{H_{0}} \frac{1}{\sqrt{2 I_{x x}(t)}}\right]^{2}+\left[\frac{H_{y}(t)}{H_{0}} \frac{1}{\sqrt{2 I_{y y}(t)}}\right]^{2}+\left[\frac{H_{z}(t)}{H_{0}} \frac{1}{\sqrt{2 I_{z z}(t)}}\right]^{2}=\frac{K(t)}{\left[H_{0}\right]^{2}}
$$

In view of Eqs. (6), this equation can be rewritten in terms of the non-dimensional angular momentum components:

$$
\left[\frac{\bar{H}_{x}}{\sqrt{2 I_{x x}(t)}}\right]^{2}+\left[\frac{\bar{H}_{y}}{\sqrt{2 I_{y y}(t)}}\right]^{2}+\left[\frac{\bar{H}_{z}}{\sqrt{2 I_{z z}(t)}}\right]^{2}=\frac{K(t)}{\left[H_{0}\right]^{2}}
$$

Finally, Eq. (12), can be written in its useful final form, used in this paper, as follows:

$$
\left[\frac{\bar{H}_{x}}{\frac{\sqrt{2 K(t) I_{x x}(t)}}{H_{0}}}\right]^{2}+\left[\frac{\bar{H}_{y}}{\frac{\sqrt{2 K(t) I_{y y}(t)}}{H_{0}}}\right]^{2}+\left[\frac{\bar{H}_{z}}{\frac{\sqrt{2 K(t) I_{z z}(t)}}{H_{0}}}\right]^{2}=1
$$

Equation (13) corresponds to the so called kinetic energy ellipsoid (KES) in the $\bar{H}_{x}, \bar{H}_{y}$ and $\bar{H}_{z}$ axis, with the following values of the semi-major axes: 

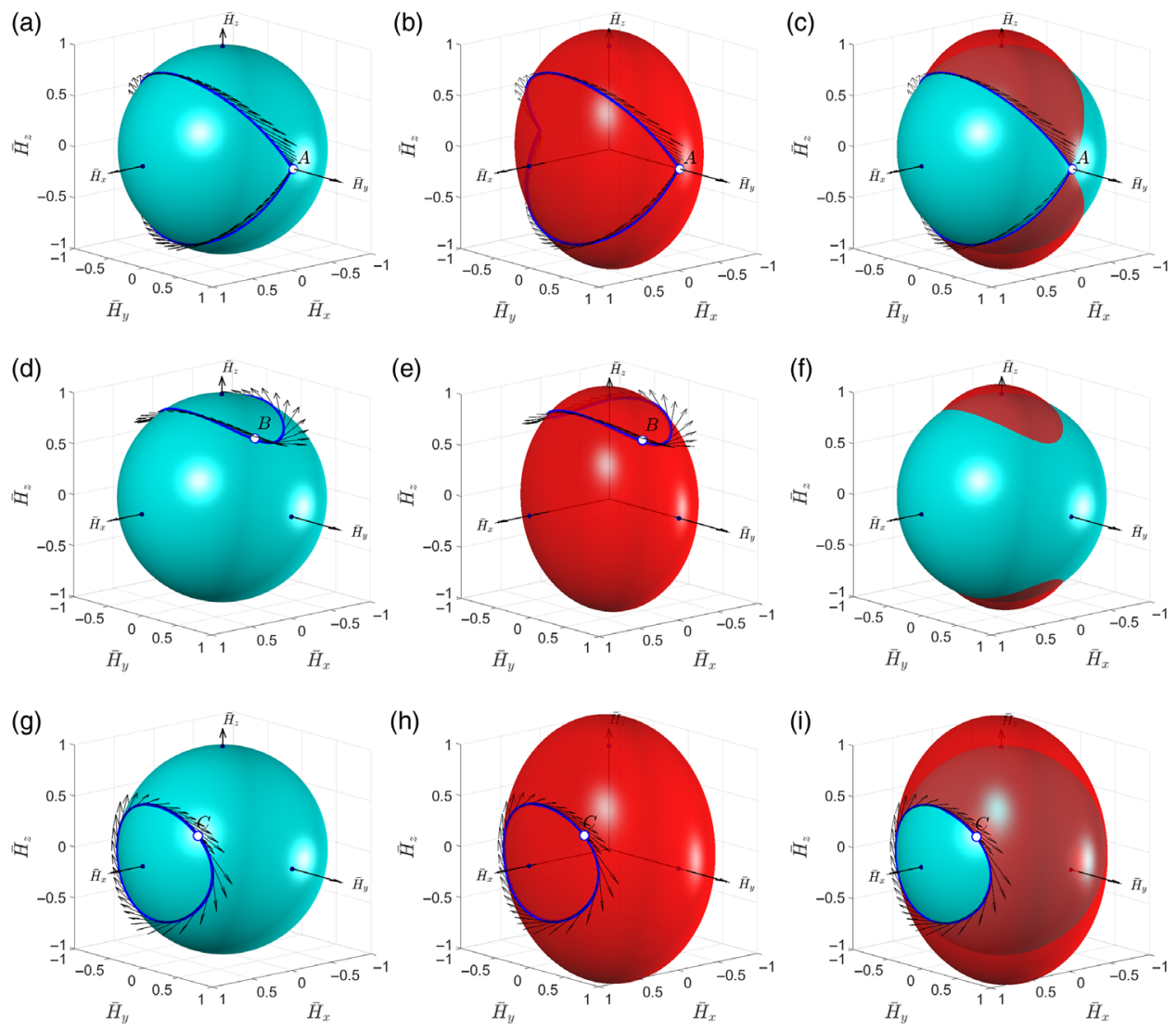

Figure 5. (a),(d),(g) Angular momentum unit spheres (left column); (b),(e),(h) kinetic energy ellipsoids (middle column) for Cases-A,B,C; (c),(f),(i) Superimposed AMSs and KEEs.

$$
a_{x}=\frac{\sqrt{2 K(t) I_{x x}(t)}}{H_{0}} ; \quad a_{y}=\frac{\sqrt{2 K(t) I_{y y}(t)}}{H_{0}} ; \quad a_{z}=\frac{\sqrt{2 K(t) I_{z z}(t)}}{H_{0}}
$$

In addition to the angular momentum spheres with specific polhodes for the cases $A, B$ and $C$ (Fig. 5, left column), let us also plot corresponding kinetic energies ellipsoids (Fig. 5, middle column). Then, combining the surfaces in these two columns, we can see that specific polhodes are, in fact, lines of intersection between corresponding AMSs and KEEs (Fig. 5, right column).

Utilising conveniences of the non-dimensional notations, we can illustrate influence of the variables $\xi$ and $\eta$ on the shapes of the kinetic energy ellipsoids and polhodes. Figure 6 presents nine contrast cases for the combinations of $\xi=[0.1,0.5,0.9]$ and $\eta=[0.2,0.5,0.9]$.

\subsection{THE DZHANIBEKOV'S EFFECT AND TENNIS RACQUET THEOREM}

The motion of the spinning rigid body, labelled as Case- $A$, has a very special significance, as it is related to the so called Dzhanibekov's Effect and Tennis Racquet Theorem ${ }^{(6)}$. Let us present a brief history of this intriguing phenomenon, partially reproduced from the reference ${ }^{(4)}$. 
(a)

$$
\eta=0.2
$$

$I_{x x}=2.00 I_{I_{y}}=2.80 I_{I_{z}}=10.00(\eta=0.200, \xi=0.100)$

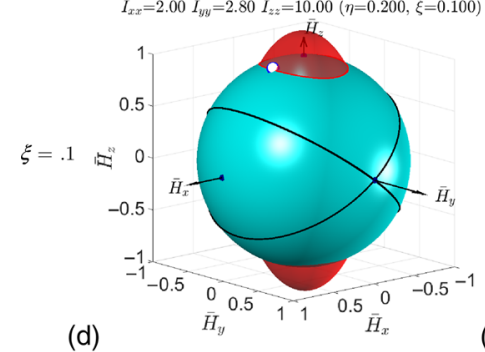

(d)

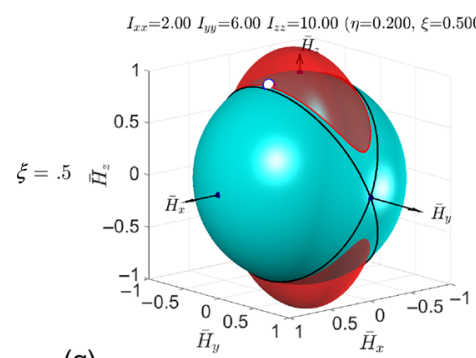

(g)

(e)

(b) $\eta=0.5$

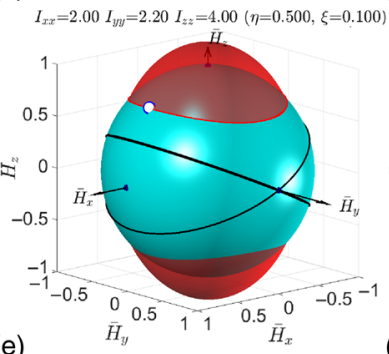

(c)

$\eta=0.9$

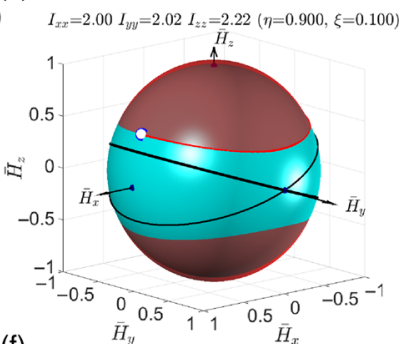

$I_{x x}=2.00 I_{y y}=3.00 I_{z z}=4.00(\eta=0.500, \xi=0.500)$
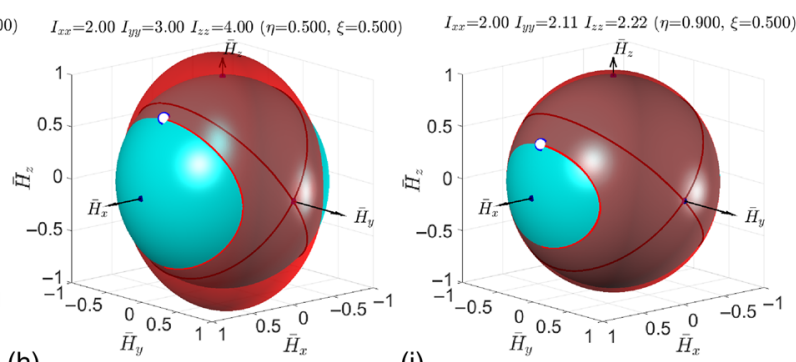

(i)

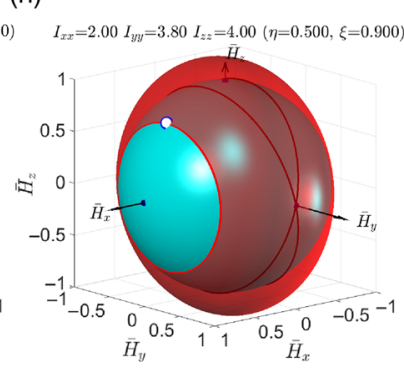

Figure 6. Contrast cases of simulations of the rotating rigid body with the same initial conditions $\left(\omega_{x 0}=\right.$ $1 ; \omega_{y 0}=0 ; \omega_{z 0}=1.5$ - all in rad $/ \mathrm{s}$ ) and $I_{x x}=2 \mathrm{~kg} \times \mathrm{m}^{2}$, illustrating changes of the shape of the kinetic energy ellipsoid due to the changes in $\eta$ and $\xi$.

Vladimir Aleksandrovich Dzhanibekov (see Fig. 7a) is one of the USSR's famous cosmonauts. During his fifth space flight, on June 25, 1985, he discovered a spectacular phenomenon: a spinning wing nut in its stable flight suddenly changed its orientation by 180 degrees and continued its flight backwards, simultaneously changing its direction of rotation to opposite! (It should be noted that wing nuts, shown in Fig. 7a and 8a, are widely used in space for fixing payloads: their shape enables removal of the wingnuts without special tools.)

Dzhanibekov realised that this pattern of motion repeated itself in the periodic sequence. Similar experiments have subsequently been run on-board of the International Space Station. Observing these experiments in space, it could be clearly seen that the spinning object always rotates in the same direction relative to the observation camera (fixed to the inertial coordinates frame): that means that in the reference frame of the rotating handle the direction of rotation flips each time its orientation flips.

Performing detailed literature search, we were able to find even earlier demonstrations in space of the flipping motion of the spinning rigid body, dated in 1973. Figure 7b shows a snapshot from the space experiment video, where famous U.S. scientist-astronaut Owen Kay 
(a)

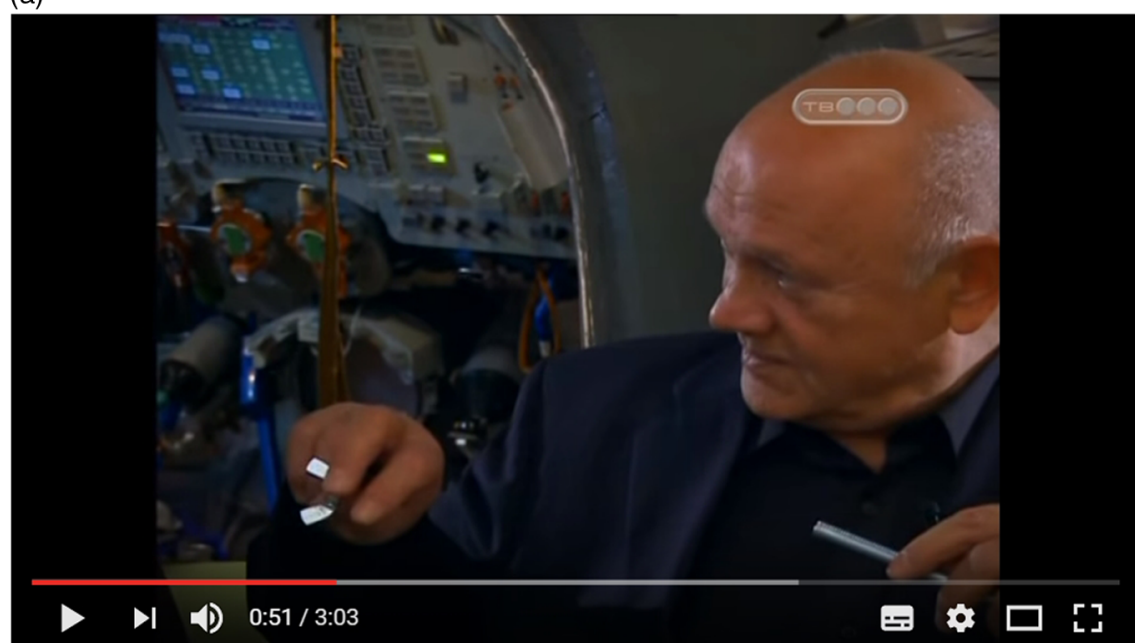

(b)

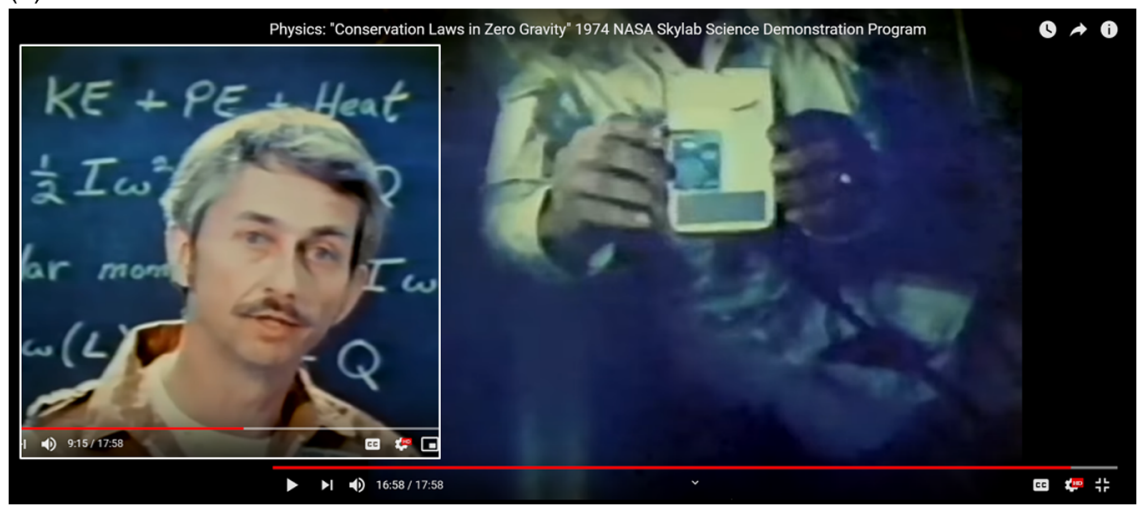

Figure 7. (a) Vladimir A. Dzhanibekov, Interview at the "Secret Signs" TV Program, explaining flipping of the wing nut, https://youtu.be/dL6Pt1O_gSE (accessed 12/10/2019); (b) Owen K. Garriott, (1973), Demonstration on board of Skylab 3 of the flipping object, spun about its intermediate axis, https://youtu.be/xdtqVR1CgQg?t=1018 (accessed 19/02/2019).

Garriott is performing a dynamics experiment onboard Skylab-3. On the snapshot, he is initiating a spin of the rigid body in zero gravity by providing an energetic torque impulse about the intermediate axis of inertia of the body, which instantly results in the peculiar rotational motion of the boxed object about this axis with clearly observed periodic flipping about this axis. Therefore, it has been documented, that the so called Dzhanibekov's Effect was observed in space in 1973 by Owen Garriott, twelve years before the same phenomenon was also observed in space in 1985 by Vladimir Dzhanibekov, whose name was given to the phenomenon.

Surprisingly, the flipping motion phenomenon, which initially was perceived by some as counter-intuitive was conceptually predicted in 1971 by Beachley ${ }^{(9)}$, however this work for very long time has been left unnoticed and popular, in-depth explanation of the phenomenon has only been very recently presented in the journal publication ${ }^{(6)}$. The Dzhanibekov's Effect 
(a)

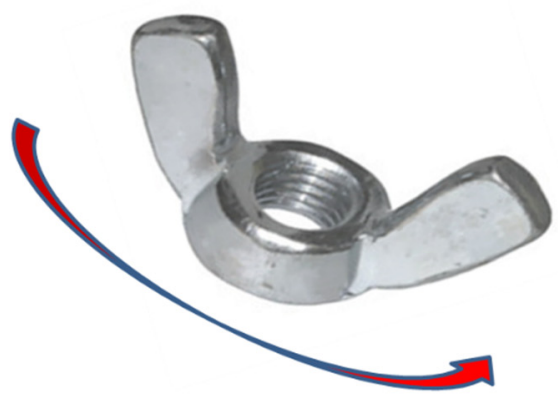

(c)

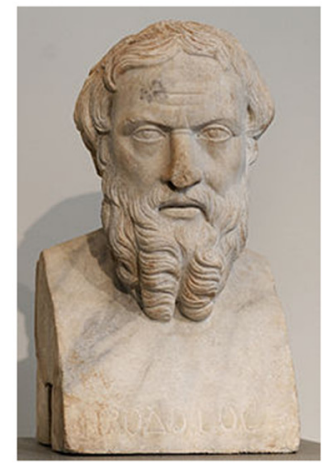

(b)

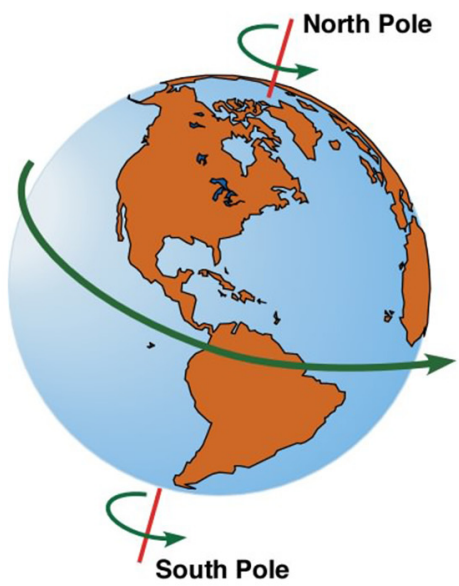

(d)

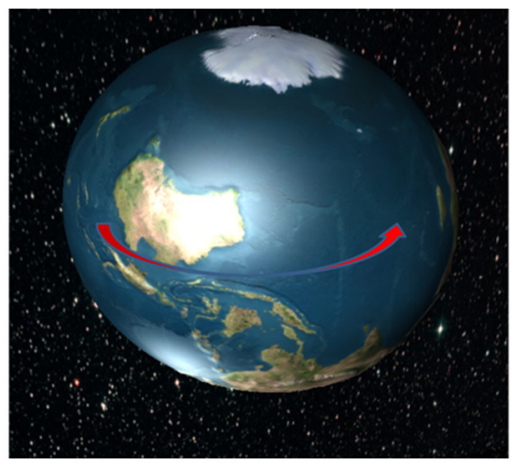

Figure 8. Is our planet, Earth, flipping similar to the wingnut? (a) simple wingnut; (b) planet Earth; (c) Herodotes, famous Greek historian; (d) imagined "flipped" Earth.

has been closely linked to the peculiar behaviour and explanation of the flipped tennis racket, which has received special names, such as Tennis Racket Theorem and intermediate axis theorem.

Explanation of both, Dzhanibekov's Effect and Tennis Racket Theorem is based on the great Euler equations, published in their canonical form in $1758^{(5)}$.

Interestingly, Euler's equations paved the theoretical ground to many scientific manifestations, including Coriolis forces, predicted by Euler, but interpreted to the world many years later by French scientist Gaspard-Gustave de Coriolis in 1835.

Entrancingly, that promotion of the Dzhanibekov's Effect has prompted development of the theories, suggesting that our planet, Earth, is performing periodic flips, similar to the wing nut (see Fig. 8d). Some researchers in the media has suggested that our planet, Earth, having much more substantial properties $\left(I \sim 8 \times 10^{37} \mathrm{~kg} \cdot \mathrm{m}^{2}\right)$, is performing these flips with much higher period, estimated to be at the order of 12,000 years. There were even some substantiation presented to justify this statement: firstly, periodicity in the changes in the magnetic field of the Earth; secondly, reference to the ancient Greeks historian, Herodotus (lived in the fifth century BC, c. 484-c. 425 BC, see Fig. 8c); and thirdly, references to the religious texts. For example, reference ${ }^{(10)}$ states: "Herodotus wrote that Egyptian priests had told him that four times since Egypt became a kingdom the Sun rose contrary to his wont; twice he rose where 
(a)

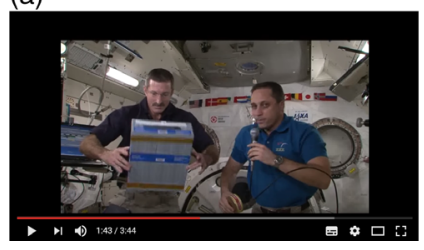

(b)

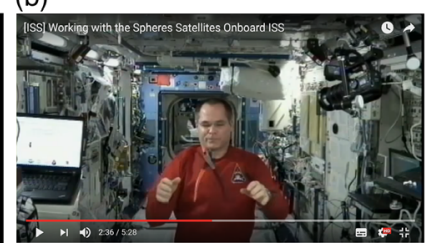

(c)

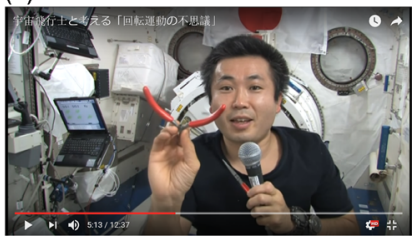

Figure 9. Demonstrations of the Dzhanibekov's Effect onboard the ISS.

(a)

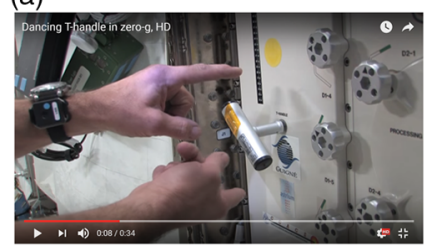

(b)

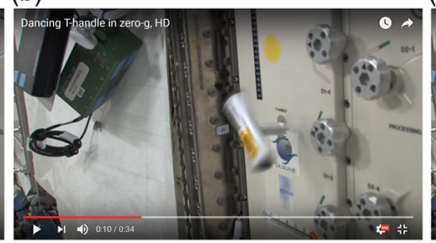

(c)

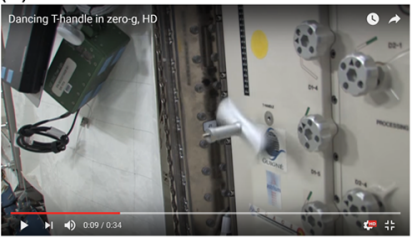

Figure 10. Dancing T-handle in zero gravity.

he now sets, and twice he set where he now rises." The Egyptians had a name for the Sun when it rose in the west, Re-Horakhty. And the concept of the Sun rising in the west occurs in both Christian and Muslim literature. There were also accounts of stars reversing the direction of rising, while various texts talk of north becoming south at a time of chaos. This reversal also appears in Greek literature, most notably in the Statesman of Plato.

The hypothesis of the flipping Earth, despite of being very intriguing, is full of controversy (for example, under conventional assumptions, the Earth is not rotating about its intermediate axis, etc.), and is not pursued further in this paper.

\subsection{DEMONSTRATIONS OF THE DZHANIBEKOV'S EFFECT ON BOARD OF THE ISS}

Due to its simplicity and intriguing nature, the Dzhanibekov's Effect has been became one of the most popular educational and scientific experiments on board of the ISS. It has been reproduced with various rigid body objects and even liquids. Various videos on these experiments, available in the media, are excellent educational resources. For example, influence of the shape of the rigid bodies, thus mass distribution in various rigid bodies, including cylinders, cubes and right rectangular prisms, was demonstrated on board of the ISS by Dan Burbank and Anton Shkaplerov (see Fig. 9a), members of thethirtieth expedition ${ }^{(11)}$.

American astronaut Kevin Ford (NASA), (thirty-fourth expedition) ${ }^{(12)}$ (see Fig. 9b) and Japanese astronaut Koichi Wakata (JAXA), (thirty-eighth expedition) ${ }^{(13)}$ (see Fig. 9c) experimented onboard the ISS with nothing more complex that pliers. They used this adjustable geometry tool as an object, capable of intriguing spinning, flipping and tumbling in zero gravity.

One of the most fascinating movies is a continuous short-period flipping of the T-handle onboard the ISS, fairly called as dancing T-handle ${ }^{(14)}$ (see Fig. 10). This is a wonderful demonstration of the Dzhanibekov's Effect, which very convincingly illustrates instability of rotation 
of the rigid body with distinct principal moments of inertia, if the main spin is provided about its principal axis, associated with intermediate moment of inertia.

All these and other demonstrations can be explained by Euler equations. Of course, this is of great interest to be able to explain interesting phenomenon of the flipping spinning systems, however, we noticed new opportunities of controlling these peculiar motions and proposed a method of control, based on the inertial morphing, involving changes of the principal moments of inertia of the system. This concept will be explained later on in the paper. However, before this, let us discuss the calculation of the period of the flipping motions in the Dzhanibekov's Effect and Tennis Racquet Theorem demos. We present some interesting results and a relevant extract from our work ${ }^{(4)}$.

\subsection{CALCULATION OF THE PERIOD OF THE FLIPPING MOTION}

As stated earlier, we assume that $I_{y y}$ is intermediate value of the principal moment of inertia. Then the period of the observed unstable motion can be estimated, using Eq. (37.12) on page 154 from the L.D.Landau reference ${ }^{(15)}$ :

$$
\text { If } H^{2}>2 K_{0} I_{y y}
$$

which is equivalent to $a_{y}<1$ (see Eq. 14), then

$$
\begin{gathered}
T=4 K \sqrt{\frac{I_{x x} I_{y y} I_{z z}}{\left(I_{z z}-I_{y y}\right)\left(H^{2}-2 K_{0} I_{x x}\right)}} \\
\text { If } H^{2}<2 K_{0} I_{y y},
\end{gathered}
$$

which is equivalent to $a_{y}>1$ (see Eq. 14), then

$$
T=4 K \sqrt{\frac{I_{x x} I_{y y} I_{z z}}{\left(I_{x x}-I_{y y}\right)\left(H^{2}-2 K_{0} I_{z z}\right)}}
$$

where $K$ is complete elliptic integral of the first kind:

$$
K=\int_{0}^{1} \frac{d s}{\sqrt{\left(1-s^{2}\right)\left(1-k^{2} s^{2}\right)}}=\int_{0}^{\pi / 2} \frac{d u}{\sqrt{1-k^{2} \sin ^{2} u}}
$$

As an illustrative example, let us assume the following parameters of the system: $I_{x x}=3, I_{z z}=$ 3.5 (all in $\left.\mathrm{kg} \times \mathrm{m}^{2}\right)$, with the initial conditions ${ }_{\mathrm{i}} \omega_{\mathrm{x}}=0.1,{ }_{\mathrm{i}} \omega_{\mathrm{y}}=15,{ }_{\mathrm{i}} \omega_{\mathrm{z}}=0.1$ (all in rad/s). For this case we will use equations (15)-(19) and will illustrate the influence of the intermediate moment of inertia $I_{y y}$ of the system on the period of the unstable flipping motion. The resulting plot, presented in Fig. 11, is clearly being asymmetrical, could be easily regarded by many as counter-intuitive, as there may be a wrongly perceived assumption of the "symmetrical" influence of $I_{y y}$ on period $T$. 


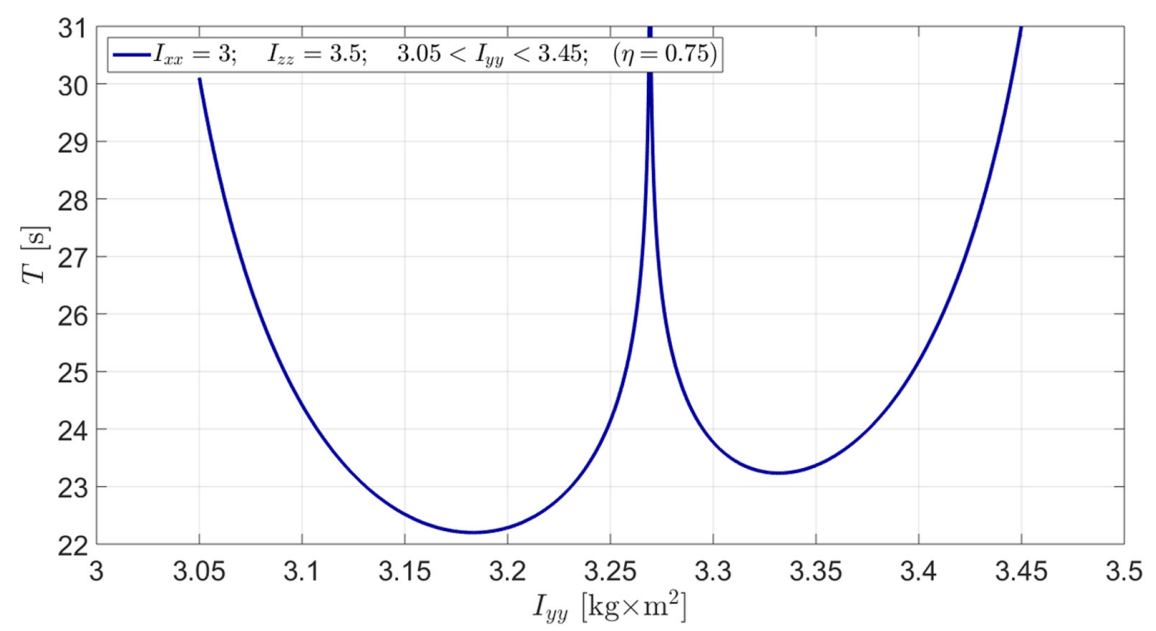

Figure 11. Period of the unstable flipping motion (Dzhanibekov's Effect case) of the rigid body, as a function of its intermediate moment of inertia $I_{y y}$ for the following example: $I_{x x}=3 ; I_{z z}=3.5\left[\mathrm{~kg} \times \mathrm{m}^{2}\right]$; $\omega_{x 0}=0.1, \omega_{y 0}=15, \omega_{z 0}=0.1[\mathrm{rad} / \mathrm{s}]$.

The plot in Fig. 11 prompts that when $I_{y y}$ is approaching any of the other moments of inertia, $I_{x x}$ or $I_{z z}$, then the period of the flipping is asymptotically approaching infinite values. Also, this plot prompts that variation in the intermediate value of the moment of inertia between $I_{x x}$ and $I_{z z}$ (i.e. changing the $\xi$ value) can allow changes of the period $T$ of the flipping motion within wide range. However, there is a minimum value of the period, which could not be reduced further. For the example shown, the lower threshold of the period is slightly higher than 22.2 seconds. Also, there is a specific value of the $I_{y y}$ that leads to the infinitely large value of the $T$. For the example shown, this corresponding value of $I_{y y}$ is approximately $3.27 \mathrm{~kg} \times \mathrm{m}^{2}$.

Similarly to the example above, in the second illustrative example, we initially assume initial conditions $\omega_{\mathrm{x} 0}=0.01, \omega_{\mathrm{y} 0}=1.5, \omega_{\mathrm{z} 0}=0.01($ all in $\mathrm{rad} / \mathrm{s})$ for the system with $I_{x x}=$ 2, $I_{z z}=4$ (all in $\mathrm{kg} \times \mathrm{m}^{2}$ ), which corresponds to $\eta=0.5$ (see Eq. 2) and plot the flipping period as a function of the intermediate moment of inertia $I_{y y}$, varying its value in-between the minimum value of the moment of inertia $I_{x x}$ and maximum value of the moment of inertia $I_{z z}$. The resultant plot is shown in Fig. 12a with a continuous red line. It allows determination of the flipping period for the Case A, illustrated previously in Figs. 1a, 2a, 5a,5b, and 5c. This value is equal to $47.16 \mathrm{~s}$, which is in agreement with Fig. 1a. Let us now, in addition to the above, consider a similar "variable $I_{y y}$ " experiment, changing only the maximum moment of inertia value from $I_{z z}=4$ to $I_{z z}=5$, which would correspond to $\eta=0.4$. The resultant plot for the period is shown with dotted blue line in Fig. 12a. Comparison of the two curves allows to suggest another avenue for manipulation with the period of the flipping motion by changing the ratio between $I_{x x}$ and $I_{z z}$, i.e. $\eta$ value.

Allowing variation of the $I_{y y}$ and $I_{z z}$ values (i.e. $\xi$ and $\eta$ non-dimensional parameters), we can also calculate more generic plot, showing influence of these principal moments of inertia on the period $T$ of the unstable motion. The resultant plot is shown in Fig. 12b. This is an interesting plot, which shows more generic nature of the asymmetry, observed in Fig. 11 and Fig. 12a. Figure 12b comprises the two curves (red and blue), presented in Fig. 12a. However, most significant observation in Fig. $12 \mathrm{~b}$ is that for each of the $I_{z z}$ values, there is a value of $I_{y y}$ 


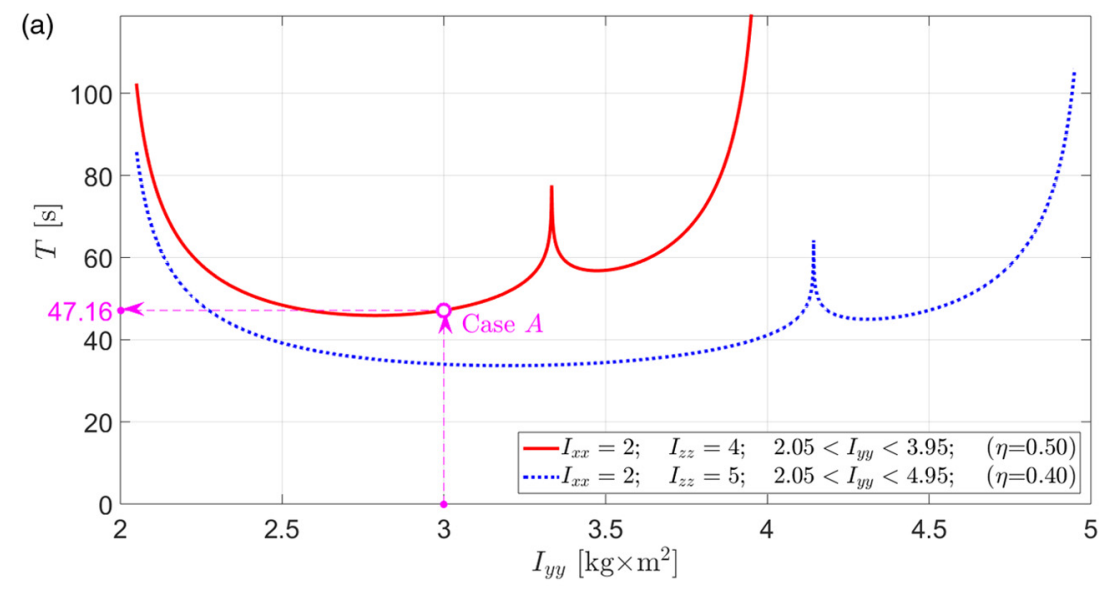

(b)

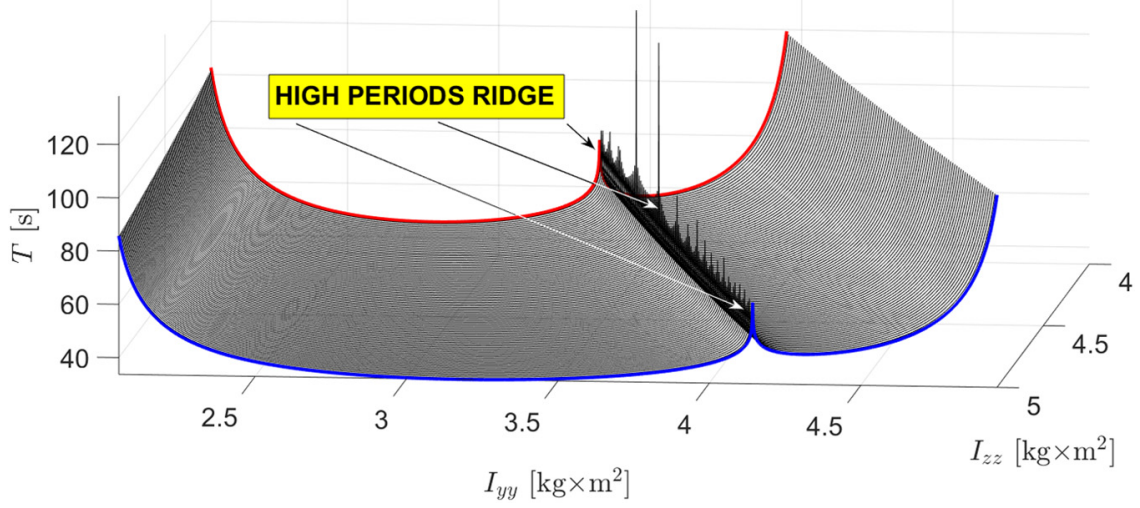

Figure 12. Period of the unstable flipping motion (Dzhanibekov's Effect case) of the rigid body, as a function of its moments of inertia $I_{y y}$ and $I_{z z}$ for two variation experiments: (a) variation of $I_{y y}$ only in two cases $I_{x x}=2 ; I_{z z}=4 ;$ and $I_{x x}=2 ; I_{z z}=4\left[\mathrm{~kg} \times \mathrm{m}^{2}\right] ; \omega_{x 0}=0.01, \omega_{y 0}=1.5, \omega_{z 0}=0.01[\mathrm{rad} / \mathrm{s}] ;$ (b) variation of both, $I_{y y}$ and $I_{z z}$ in the case $I_{x x}=2\left[\mathrm{~kg} \times \mathrm{m}^{2}\right] ; \omega_{x 0}=0.01, \omega_{y 0}=1.5, \omega_{z 0}=0.01[\mathrm{rad} / \mathrm{s}]$.

which leads to the infinitely large period of the flipping motion. We named this area as "high periods ridge", clearly labelled in Fig. 12b.

\subsection{CONCEPT OF THE INERTIAL MORPHING OF THE SPACECRAFT}

As an enhancement in the control capabilities of the spacecraft, in our previous works ${ }^{(1-5)}$, we proposed a concept of inertial morphing: we showed that using special devices (with, for example moving masses) or other means and/or phenomena, (for example, moving liquids, mass evaporation, solidification, ablation), enabling controlled modifications of the principle moments of inertia characteristics, the attitude dynamics of the spacecraft could be efficiently controlled.

Assume that the spacecraft has morphing capabilities, allowing independent controllable changes of the values of the principal moments of inertia. A basic model of the morphing spacecraft, involving three orthogonal dumbbells, each of which has negligible mass of 


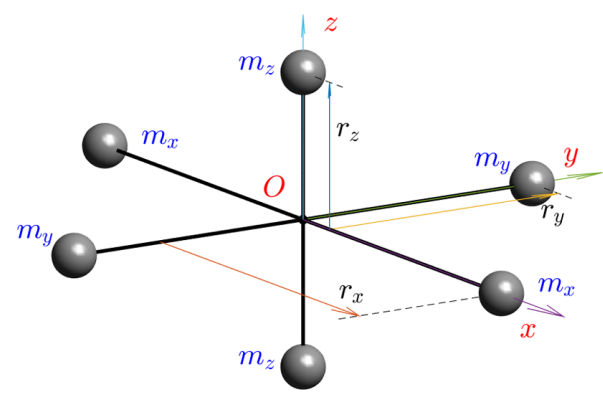

Figure 13. Six-mass conceptual model of the morphing spacecraft.

the rod, connecting two equal concentrated masses at its ends, was considered in ${ }^{(5)}$ and is reproduced in Fig. 13.

Let us also assume, for conceptual simplicity, that three dumbbells are connected at the middle points of their rods, and the corresponding masses $m_{x}, m_{y}$ and $m_{z}$ are located at the distances $r_{x}, r_{y}$ and $r_{z}$ from the axes of rotation $x, y$ and $z$, as shown in Fig. 13. In the illustrated conceptual design, morphing of the spacecraft is achieved via independent synchronized control of the position coordinates $r_{x}=r_{x}(t), r_{y}=r_{y}(t)$ and $r_{z}=r_{z}(t)$ of the masses $m_{x}, m_{y}$ and $m_{z}$.

Indeed, changing the distance between three pairs of the masses could be used to achieve any values of the principal moments of inertia $I_{x x}, I_{y y}$ and $I_{z z}$. To achieve this objective, it would be sufficient to move masses to the following radii:

$$
r_{x}=\sqrt{\frac{I_{y y}+I_{z z}-I_{x x}}{4 m_{x}}} ; \quad r_{y}=\sqrt{\frac{I_{z z}+I_{x x}-I_{y y}}{4 m_{y}}} ; \quad r_{z}=\sqrt{\frac{I_{x x}+I_{y y}-I_{z z}}{4 m_{z}}}
$$

These important relationships can be easily obtained from the equations for principal moments of inertia of the system ${ }^{(16)}$ :

$$
I_{x x}=2 m_{y} r_{y}^{2}+2 m_{z} r_{z}^{2} ; \quad I_{y y}=2 m_{z} r_{z}^{2}+2 m_{x} r_{x}^{2} ; \quad I_{z z}=2 m_{x} r_{x}^{2}+2 m_{y} r_{y}^{2}
$$

\subsection{SUGGESTIONS ON SOME PRACTICAL IMPLEMENTATION OF THE INERTIAL MORPHING}

This paper does not aim to present comprehensive collection of the implementation of the methods to control the principal moments of inertia of the spacecraft, we called "inertial morphing". Nevertheless, for completeness, we wish to present just a few of the methods/concepts, being considered as promising for realization in real spacecraft systems. In these examples, for simplicity of the illustrations, the conceptual model of the spacecraft (Fig. 13) will be used. As equations for the moments of inertia are functions of the distances $r$ and masses $m$, conceptually, there could be two main approaches to the implementation of the inertial morphing: (a) based on variation of $r$ - positions of the masses and (b) based on variation of masses. These approaches are briefly explained below.

(a) The first approach to implementation of the inertial morphing is based on the controlled re-position of the spacecraft masses, using actuators. Let us consider the following case: $m_{x}=m_{y}=m_{z}=1 \mathrm{~kg}$; these masses are initially located at their radii: $r_{x}=0.8 \mathrm{~m}$; 
(a)

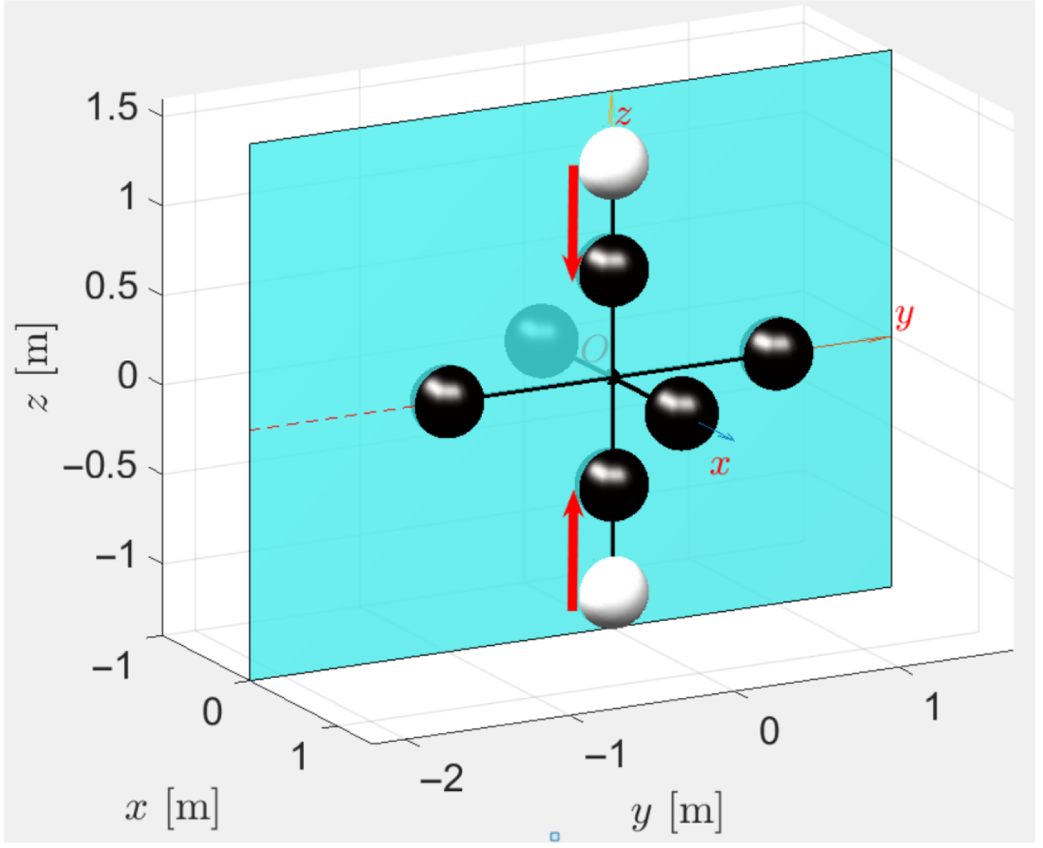

(b)

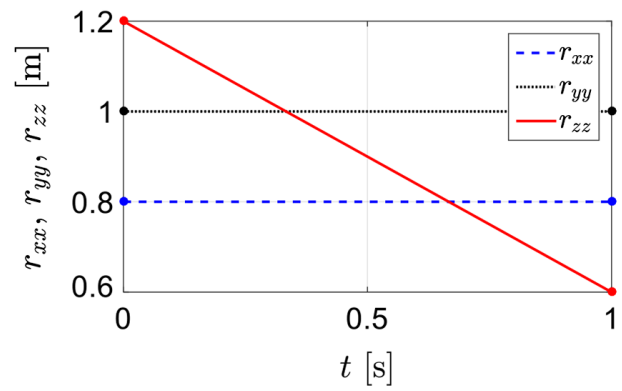

(c)

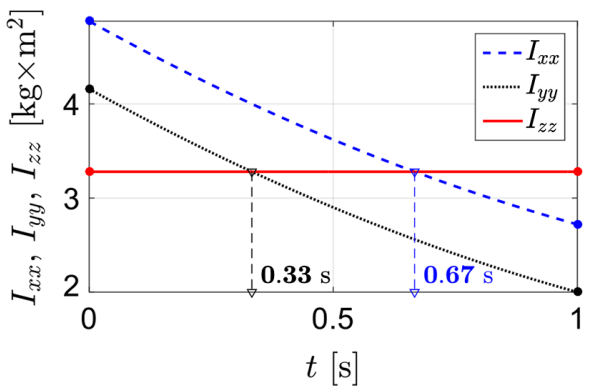

Figure 14. Particular example of inertial morphing via translational re-position of the $z$ dumbbell masses $\mathrm{m}_{z}$, while keeping positions of the $\mathrm{x}$ and $\mathrm{y}$ masses unchanged $\left(m_{x}=m_{y}=m_{z}=1 \mathrm{~kg}\right)$ : (a) 3D view of the spacecraft model; (b) time history of the position of the masses; (c) time history of the resulting principal moments of inertia $I_{x x}, I_{y y}$ and $I_{z z}$.

$r_{y}=1 \mathrm{~m} ; r_{z}=1.2 \mathrm{~m}$. Let us assume that the system is equipped with linear actuator (motor and appropriate mechanical system), capable of translational re-positioning of the masses $m_{z}$ via changing the length of $r_{z}$ from $1.2 \mathrm{~m}$ to $0.6 \mathrm{~m}$ within $1 \mathrm{~s}$. The morphing process is shown in Fig. 14a, where initial positions of the masses are shown with white spheres, and the final positions - with black spheres and where direction of the translations for two $m_{z}$ masses is shown with two red arrows. Also, for better perception of the $3 \mathrm{D}$ design, a semi-transparent $y z$ plane is added to Fig. 14a.

In this example, the positions of the masses on the $x$ and $y$ axes remain unchanged, only $r_{z}$ is subject to variation (as per Fig. 14b). Equation. (21) permits the calculation of the associated resulting time history of the principle moments of inertia of the system. Figure 14c shows that 


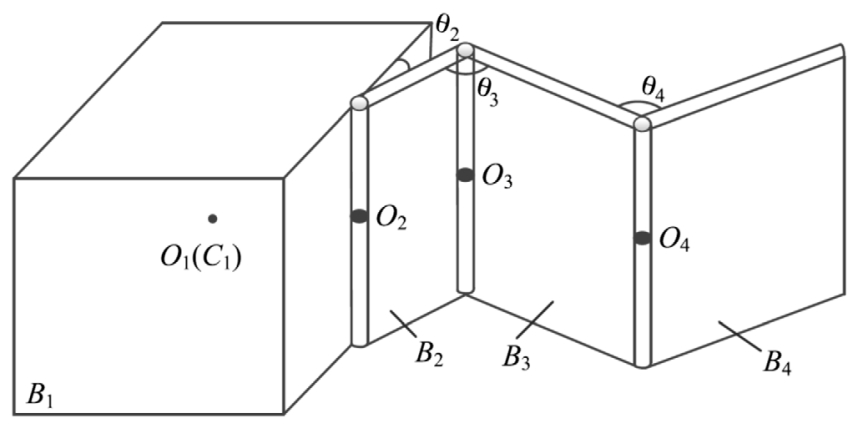

Figure 15. Spacecraft, deploying solar $\operatorname{arrays}^{(17)}$.

while $I_{z z}$ keeps its value unchanged, during morphing, the $I_{x x}$ value is changing from 4.88 to $2.72\left[\mathrm{~kg} \times \mathrm{m}^{2}\right]$ and the $I_{y y}$ is changing from 4.16 to $2.0\left[\mathrm{~kg} \times \mathrm{m}^{2}\right]$. However, most significant in the context of this paper is an observation that, in this example, the role of the intermediate moment of inertia (which initially "belongs" to $I_{y y}$ ) is "passed" from $I_{y y}$ to $I_{z z}$ (at $t=0.33 \mathrm{~s}$ ) and then is further passed to $I_{x x}$ (at $t=0.67 \mathrm{~s}$ ). Consequently, using only one variable $r_{z}$ in the morphing process, it was possible to arrange for each of the spacecraft axes $x, y$ and $z$ at different stages, to become the intermediate axis of rotation.

The method presented above can be extended to the actuation of all masses, including $m_{x}$ and $m_{y}$. With this general arrangement, the morphing would permit continuous control of the position of all masses, hence, would enable assignment of any arbitrary values to the principal moments of inertia of the system, as per requirements of the morphing scenarios. Of course, these assignments should be compatible with the mechanical/electrical/thermal constrains of the particular designs/implementations of the morphing systems.

A variation of the same method may involve application of the special actuators to reposition large segments of the spacecraft. This idea is illustrated with the controlled change of the angular positions $\theta_{i}$ of the solar panels to manipulate the inertial properties of the spacecraft (see Fig. 15).

We envisage that similar implementations of the illustrated principle can be achieved in some other ways. For example, deployment of the masses, to the new destination (in any, inwards, outwards or inclined directions) can be ensured via un-constraining the precompressed springs, as per Fig. 16. This, however, would permit only a single discrete actuation.

Alternatively, for continuous actuation, instead of using solid masses, heavy liquids and/or liquid metals ${ }^{(18)}$ can be used, which could be controlled via manipulation with valves and employment of the passive inertial forces and/or controlled magnetic field forces to move these liquid medias.

(b) The second approach to implementation of the inertial morphing is based on the controlled change of the spacecraft masses and may involve, for example, mass ejection; ablation, evaporation or solidification of the components of the structure, etc.

Geometric reconfigurations of the spacecraft systems (for example, during deployment of the inflatable components or solar panels, and reorientation of the antennae) is a widely used concept and proved to be successful for many space systems (for example, Spartan-207, Hughes/Boeing HS-376, SMART-1 and RAE-B satellites, space probes Rosetta and Down, etc.). However, these are provided to ensure the functionality of the spacecraft, without any 
(a)

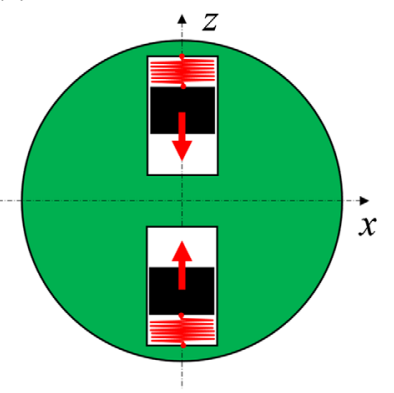

(b)

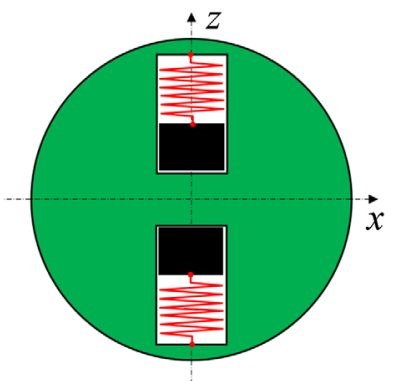

Figure 16. Particular example of inertial morphing via translational re-position of the $z$ dumbbell masses $\mathrm{m}_{\mathrm{z}}$, (shown with black color) via release of the pre-constrained compressed springs (shown with red color): (a) initial configuration; (b) masses deployed inwards.

relevance to the attitude dynamics objectives ${ }^{(19)}$. In contrast, concept of spacecraft reconfiguration, explicitly aiming to assist in attitude maneuvering ${ }^{(1-5)}$, is a very new concept. Indeed, idea of the reconfigurable spacecraft systems, transformable spacecraft, which consist of multiple modules connected with each other by hinges or universal joints, proposed by $\operatorname{JAXA}^{(20,21)}$, is only one year old.

It is believed that this paper will further contribute to a much wider application of the spacecraft reconfigurations with the primary goal to enhance the attitude dynamics capabilities of spacecraft systems, especially in view of a new findings, presented in the following sections.

\subsection{NUMERICAL SIMULATION OF THE MORPHING SYSTEMS}

To simulate the spacecraft systems with morphing capabilities, the Euler equations must be modified to treat the principle moments of inertia not as constants (assumed in the classical Euler equations), but as variables:

$$
\left[\begin{array}{ccc}
\dot{I}_{x x} & 0 & 0 \\
0 & \dot{I}_{y y} & 0 \\
0 & 0 & \dot{I}_{z z}
\end{array}\right]\left\{\begin{array}{l}
\omega_{x} \\
\omega_{y} \\
\omega_{z}
\end{array}\right\}+\left[\begin{array}{ccc}
I_{x x} & 0 & 0 \\
0 & I_{y y} & 0 \\
0 & 0 & I_{z z}
\end{array}\right]\left\{\begin{array}{l}
\dot{\omega}_{x} \\
\dot{\omega}_{y} \\
\dot{\omega}_{z}
\end{array}\right\}+\left[\begin{array}{ccc}
0 & -\omega_{z} & \omega_{y} \\
\omega_{z} & 0 & -\omega_{x} \\
-\omega_{y} & \omega_{x} & 0
\end{array}\right]\left[\begin{array}{ccc}
I_{x x} & 0 & 0 \\
0 & I_{y y} & 0 \\
0 & 0 & I_{z z}
\end{array}\right]\left\{\begin{array}{l}
\omega_{x} \\
\omega_{y} \\
\omega_{z}
\end{array}\right\}=\left\{\begin{array}{l}
0 \\
0 \\
0
\end{array}\right\}
$$

These equations can be bundled with quaternions or Euler angles relationships. The version from $^{(5)}$ is presented below:

$$
\left[\begin{array}{cccccc}
I_{x x} & 0 & 0 & 0 & 0 & 0 \\
0 & I_{y y} & 0 & 0 & 0 & 0 \\
0 & 0 & I_{z z} & 0 & 0 & 0 \\
0 & 0 & 0 & \sin \theta \sin \phi & \cos \phi & 0 \\
0 & 0 & 0 & \sin \theta \cos \phi & -\sin \phi & 0 \\
0 & 0 & 0 & \cos \theta & 0 & 1
\end{array}\right]\left\{\begin{array}{c}
\dot{\omega}_{x} \\
\dot{\omega}_{y} \\
\dot{\omega}_{z} \\
\dot{\psi} \\
\dot{\theta} \\
\dot{\phi}
\end{array}\right\}=\left\{\begin{array}{c}
\left(I_{y y}-I_{z z}\right) \omega_{y} \omega_{z}-\dot{I}_{x x} \omega_{x} \\
\left(I_{z z}-I_{x x}\right) \omega_{z} \omega_{x}-\dot{I}_{y y} \omega_{y} \\
\left(I_{x x}-I_{y y}\right) \omega_{x} \omega_{y}-\dot{I}_{z z} \omega_{z} \\
\omega_{x} \\
\omega_{y} \\
\omega_{z}
\end{array}\right\}
$$

These equations have the following "mass matrix" format:

$$
[M(t, \mathbf{x})]\{\dot{\mathbf{x}}\}=\{f(t, \mathbf{x})\}
$$


(a)

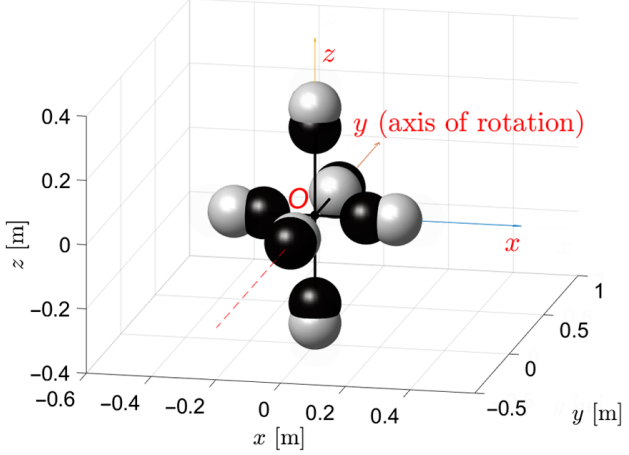

Solution-1 (b)

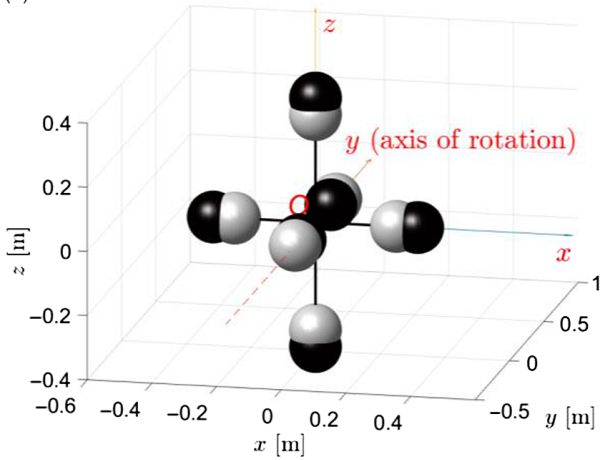

Solution-2

Figure 17. Graphical representation of solutions for stopping flipping motion. White spheres - initial unstable configuration for $y$ main rotation, black spheres - final stable configuration.

and can be solved numerically, using, for example, ode MATLAB ${ }^{\circledR}$ Runge-Kutta solver, with "mass matrix" option.

\subsection{BASIC DEMONSTRATION OF THE INERTIAL MORPHING CAPABILITIES: STOPPING FLIPPING MOTION IN THE DZHANIBEKOV'S EFFECT DEMO [2]}

Let us assume, for illustration purposes, that $m_{x}=m_{y}=m_{z}=1 \mathrm{~kg}, I_{x x}=0.3 \mathrm{~kg} \times \mathrm{m}^{2}, I_{y y}=$ $0.35 \mathrm{~kg} \times \mathrm{m}^{2}, I_{z z}=0.4 \mathrm{~kg} \times \mathrm{m}^{2}$. Then, equations (20) would enable us to determine the exact values of the initial radii $r_{x}, r_{y}$ and $r_{z}$, compatible with the requirements for the $I_{x x}, I_{y y}, I_{z z}$ values:

$$
r_{x}=0.2500 \mathrm{~m} ; r_{y}=0.2958 \mathrm{~m} ; r_{z}=0.3354 \mathrm{~m}
$$

Note that in our example here $I_{y y}$ has an intermediate value among all principal moments of inertia: $I_{x x}<I_{y y}<I_{z z}$, therefore if the spacecraft is provided with the initial angular velocities $\omega_{x}=\omega_{z}=0.1 \mathrm{rad} / \mathrm{s}$ and $\omega_{y}=15 \mathrm{rad} / \mathrm{s}$, with the prevailing rotation about $y$ body axis, then the spacecraft rotation about this axis would be unstable and classical Dzhanibekov's Effect periodic flipping would be observed.

Let us during the flipping motion, at the instant, when the angular velocities $\omega_{x}$ and $\omega_{z}$ are close to zeros, rapidly change the moment of inertia $I_{y y}$ to its new value of f $I_{y y}=0.2 \mathrm{~kg} \times \mathrm{m}^{2}$.

Then the moment of inertia $I_{y y}$ stops being the intermediate value, and the rotation about $y$ body axis would becoming stable, without changes in the direction of $\omega_{y}$.

It has been demonstrated in ${ }^{(2,5)}$ that there are two classes of solutions. The new values of the position radii, corresponding to the "solution-1", can be calculated using Eq. (20):

$$
r_{x}=0.1581 \mathrm{~m} ; r_{y}=0.3536 \mathrm{~m} ; r_{z}=0.2739 \mathrm{~m}
$$

The spacecraft masses at these radius positions are shown in Fig. 17(a) with dark color. 
Table 1

Numerical values of the solutions for stopping flipping motion

Solution-1

$\begin{array}{lcccccc} & \boldsymbol{r}_{\boldsymbol{x}} & \boldsymbol{r}_{\boldsymbol{y}} & \boldsymbol{r}_{\boldsymbol{z}} & \boldsymbol{I}_{\boldsymbol{x} \boldsymbol{x}} & \boldsymbol{I}_{\boldsymbol{y} \boldsymbol{y}} & \boldsymbol{I}_{\boldsymbol{z} z} \\ \text { Initial } & 0.2500 & 0.2958 & 0.3354 & 0.30 & \mathbf{0 . 3 5} & 0.40 \\ \text { Final } & 0.1581 & 0.3536 & 0.2739 & 0.30 & \mathbf{0 . 2 0} & 0.40\end{array}$

\section{Solution-2}

$\begin{array}{lcccccc} & \boldsymbol{r}_{\boldsymbol{x}} & \boldsymbol{r}_{\boldsymbol{y}} & \boldsymbol{r}_{\boldsymbol{z}} & \boldsymbol{I}_{\boldsymbol{x} \boldsymbol{x}} & \boldsymbol{I}_{\boldsymbol{y} \boldsymbol{y}} & \boldsymbol{I}_{\boldsymbol{z} \boldsymbol{z}} \\ \text { Initial } & 0.2500 & 0.2958 & 0.3354 & 0.30 & \mathbf{0 . 3 5} & 0.40 \\ \text { Final } & 0.3162 & 0.2236 & 0.3873 & 0.30 & \mathbf{0 . 5 0} & 0.40\end{array}$

(a)

(b)

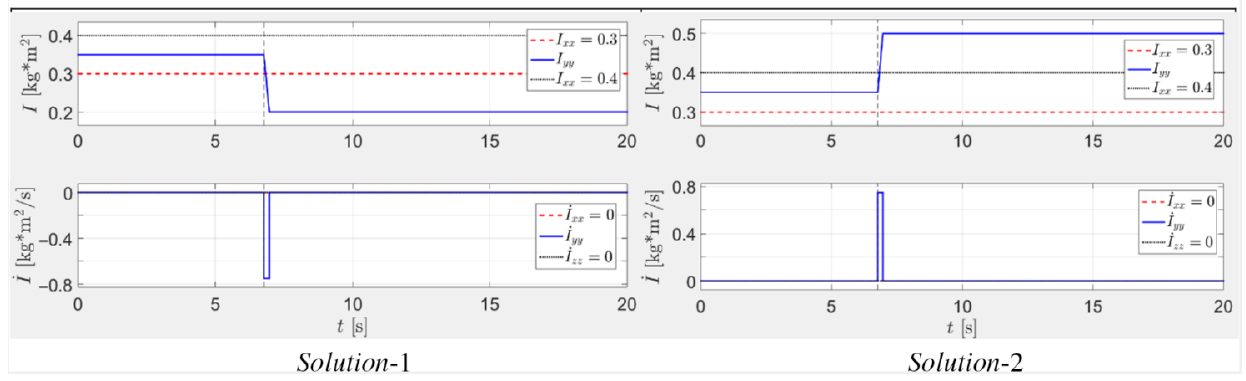

Figure 18. Graphical representation of solutions for stopping flipping motion: time histories of the required controlled manipulation with the moment of inertia $I_{y y}$.

The flipping motion can be also stopped using "solution-2". For the purpose of the illustration of the concept, let us consider rapid increase of the $I_{y y}$ from its initial value of $0.35 \mathrm{~kg} \times \mathrm{m}^{2}$ to its new value of $0.5 \mathrm{~kg} \times \mathrm{m}^{2}$. The new values of the position radii, corresponding to the "solution-2" can be calculated, using Eq. (20):

$$
r_{x}=0.3162 \mathrm{~m} ; r_{y}=0.2236 \mathrm{~m} ; r_{z}=0.3873 \mathrm{~m} .
$$

The spacecraft masses at these radius positions are shown in Fig. 17(b) with dark color.

The morphing of the spacecraft from the initially unstable configuration, associated with the flipping motion, to its final stable configuration and Solution 1 and 2 are shown in Fig. 17, where masses for the initial configuration are shown in white, whereas the masses for the final configuration are shown in black color. Summary for both solutions is presented in Table 1. It would be important to note, that in the presented cases, it was not obligatory during the morphing of the system and its transition from the "initial" to "final" states to keep both values of $I_{x x}$ and $I_{z z}$ unchanged. However, it was done for purpose, to emphasize the role of the $I_{y y}$ in the process of stabilisation of the system.

Results of the corresponding numerical simulations of these two solutions are presented in Fig. 18-20. 
(a)

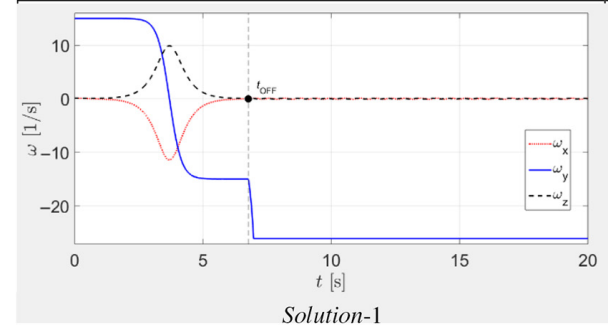

(b)

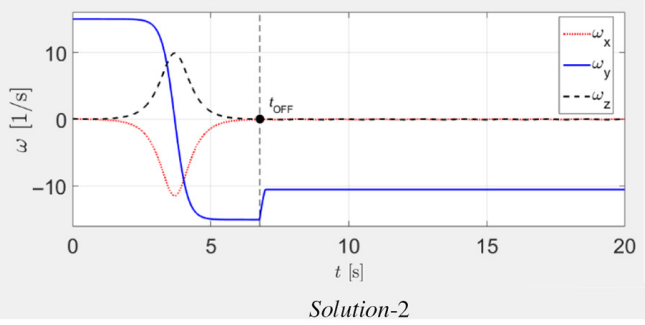

Figure 19. Graphical representation of solutions for stopping flipping motion: time histories of the $\omega_{x}, \omega_{y}, \omega_{z}$.

(a)

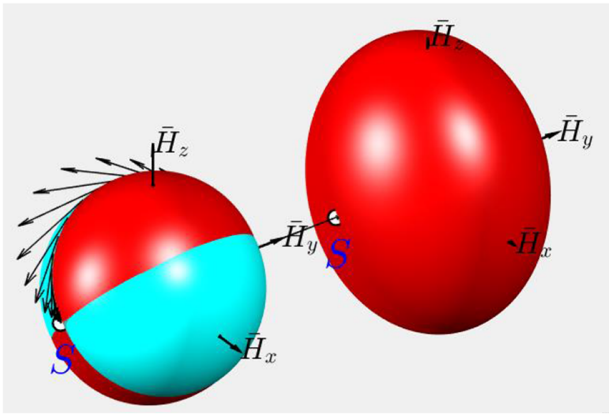

Solution-1 (b)

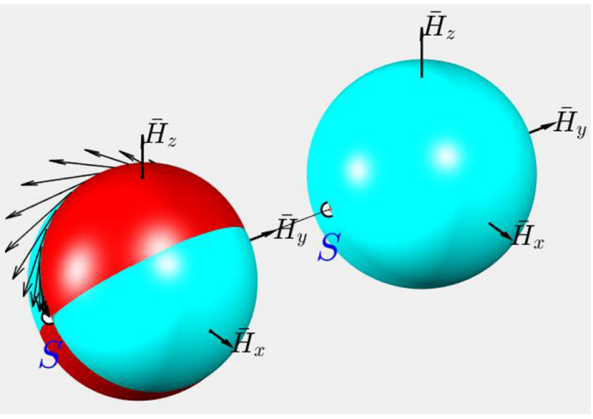

Solution-2

Figure 20. Graphical interpretation of solutions for stopping flipping motion.

It is interesting to note, that with Solution1, stabilisation of the spinning body is achieved via expansion of the kinetic energy ellipsoid, which completely embraces the angular momentum sphere (see Fig. 20a). On the right part of the Fig. 20a, both surfaces are just only touching each other at the point $\mathrm{S}$ and on the opposite side of the $\mathrm{y}$-axis.

However, with Solution-2, stabilisation of the spinning body is achieved via shrinking of the kinetic energy ellipsoid, which becoming completely embraced by the angular momentum sphere (see Fig. 20b). On the right part of the Fig. 20b, both surfaces are just only touching each other at the point $\mathrm{S}$ and on the opposite side of the $\mathrm{y}$-axis.

\subsection{INVESTIGATING ORIENTATION OF THE SIDES OF THE SPACECRAFT, EXPOSED TO THE SPECIFIC DIRECTIONS}

As spacecraft may have directional sensing equipment, attached to the sides, let us explore possible exposure of this equipment to the specified directions of interest. For this purpose let us introduce a semi-transparent green coloured spherical "dome", embracing the rotating spacecraft (which, in turn, has its rotating body axes system $x y z$ with unit orts $\mathbf{e}_{1} \mathbf{e}_{2}$ and $\mathbf{e}_{3}$ ). We collocate the centre of the dome (point $O$ ) with the centre of the mass of the rotating body. However, most significant, we fix the dome in the global coordinates $X Y Z$, so is not rotating with the body and its body axes $x y z$. Then we consecutively plot lines of intersection of the 
(a)
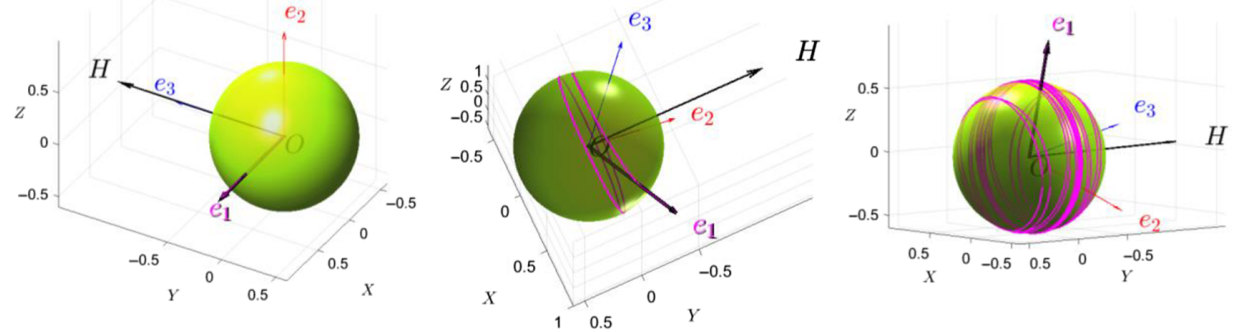

(b)

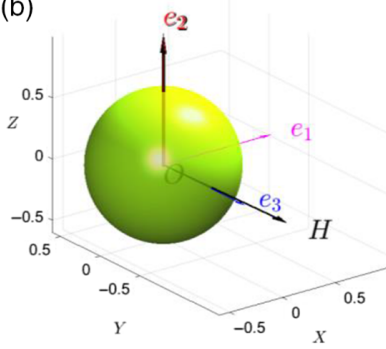

$\mathbf{e}_{1}$ diagrams $(t=0,12$ and $120 \mathrm{sec})$
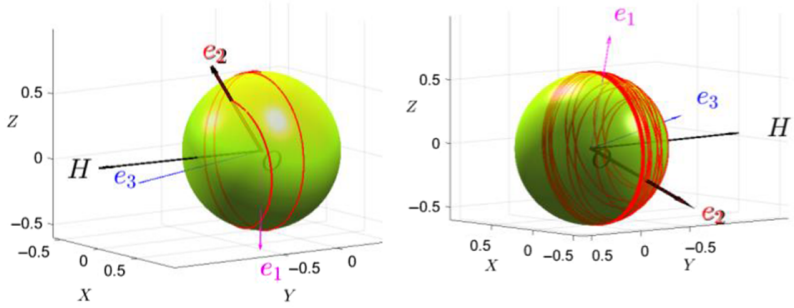

$\mathbf{e}_{2}$ diagrams $(t=0,12$ and $120 \mathrm{sec})$
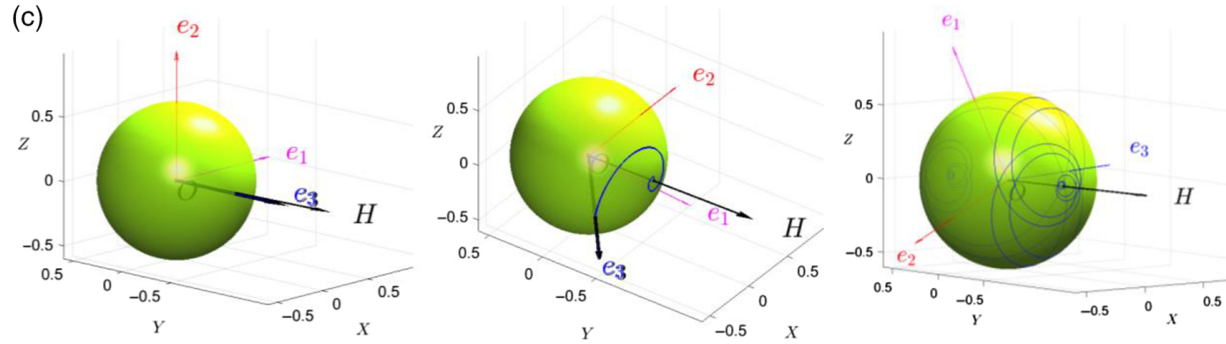

(d)

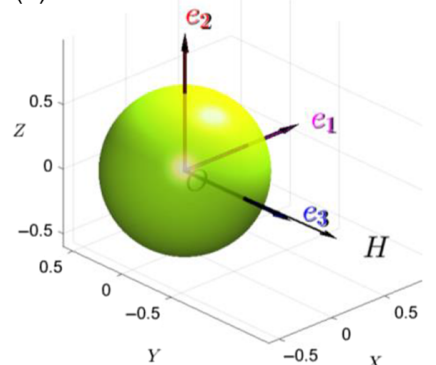

$\mathbf{e}_{3}$ diagrams $(t=0,12$ and $120 \mathrm{sec})$
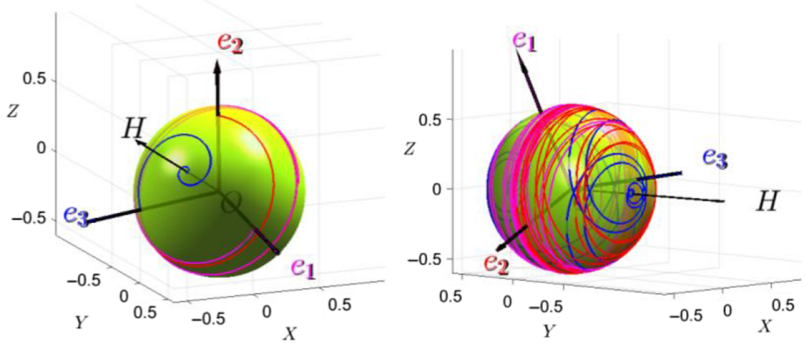

$\mathbf{e}_{1}, \mathbf{e}_{2}$ and $\mathbf{e}_{3}$ diagrams, presented together $(t=0,12$ and $120 \mathrm{sec})$

Figure 21. Lines of intersection of the rotating orts $\mathbf{e}_{1} \mathbf{e}_{2}$ and $\mathbf{e}_{3}$ with the spherical dome (green), fixed in the global axes system $X Y Z$ : ball-of-wool lines.

rotating orts $\mathbf{e}_{1} \mathbf{e}_{2}$ and $\mathbf{e}_{3}$ with the dome. It must be emphasised, that the spheres in Fig. 21 are not the bodies of the spacecraft (which may have any arbitrary shape), but the embracing imaginary domes.

For the illustration purposes, let us simulate the motion of the spacecraft with the following parameters: $I_{x x}=2, I_{y y}=4, I_{z z}=3$ (all in $\mathrm{kg} \times \mathrm{m}^{2}$ ). Let us for $t=0$ align $x y z$ body axes 


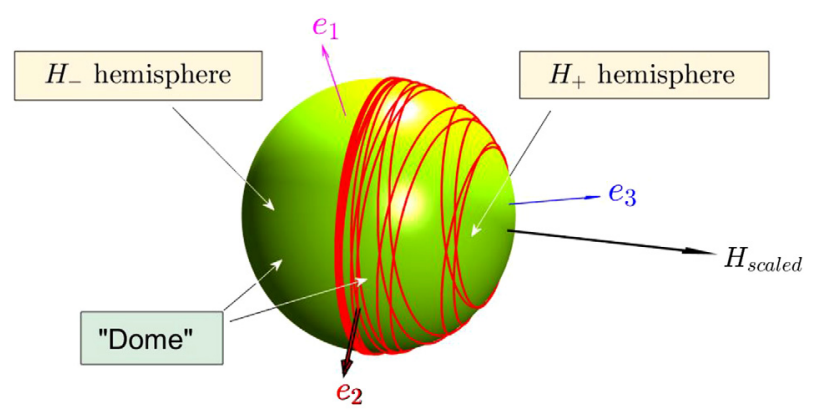

Figure 22. $H_{+}$and $H$. hemispheres of the dome $\left(I_{x x}=2, I_{y y}=4, I_{z z}=3\right.$, all in $k g \times m^{2} ; \omega_{x}=0.01, \omega_{y}=0.01$, $\omega_{z}=1$, all in rad/s).

with $X Y Z$ global inertial axes as follows: $x$ is aligned with $X, y$ is aligned with $Z, z$ is aligned with $-Y$. If the spacecraft is installed in orbit with initially provided angular velocities $\omega_{x 0}=$ $0.01, \omega_{y 0}=0.01, \omega_{z 0}=1$ (all in $\left.\mathrm{rad} / \mathrm{s}\right)$, the spacecraft starts flipping along axis $z$, being an intermediate axis of inertia (as $I_{x x}<I_{z z}<I_{y y}$ ).

During this flipping process we trace all intersections of the orts $\mathbf{e}_{1} \mathbf{e}_{2}$ and $\mathbf{e}_{3}$ with the dome and present them as continuous lines with different colors. Results are shown in Fig. 21. It should be noted, that for each of the computer screen snap-shots in this figure, the individual viewpoint was selected for better observation of the simulation results. Selection of the viewpoints could be clearly understood using the vector of the angular momentum $\mathbf{H}$ as a reference, as it is pointing in the same direction in the global coordinates $X Y Z$ for all presented snap-shots.

Last image for the $\mathbf{e}_{2}$ in the middle row in Fig. 22 is remarkably interesting and illustrates our new finding! It shows that $y$ body rotating axis, associated in this example with the maximum moment of inertia, is drawing $\mathbf{e}_{2}$ intersection lines on the dome only on the hemisphere, bulging towards the angular momentum vector $\mathbf{H}$ (we call it $H_{+}$hemisphere) and is never

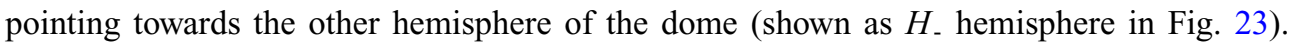
This is valid for the direction of $y$ with positive component of the angular velocity along this direction $\left(\omega_{y 0}>0\right)$. We have run many other various simulations, confirming that it is a general pattern, so the side, perpendicular to the axis with maximum moment of inertia and associated with positive angular velocity component, is never turned away from the vector $\mathbf{H}$ direction.

In Fig. 21, initially, vector $\mathbf{H}$ is almost aligned with $z$ body axes (which is, in turn, is initially positioned along the $-Y$ global axis), this is because initial values of $\omega_{x 0}$ and $\omega_{z 0}$ (and ultimately $H_{x 0}$ and $H_{z 0}$ ) are small compared with $\omega_{y 0}$ (and ultimately $H_{y 0}$ ). Therefore, the 2D plane surface, subdividing $H_{+}$and $H_{-}$is almost parallel to the $X Z$ plane. $H_{+}$and $H_{-}$are also shown in Fig. 22.

Let us consider additional contrast case with the following parameters: $I_{x x}=2, I_{y y}=4$, $I_{z z}=3$ (all in $\mathrm{kg} \times \mathrm{m}^{2}$ ) and initial angular velocities $\omega_{x 0}=0.5, \omega_{y 0}=0.5, \omega_{z 0}=1$ (all in $\mathrm{rad} / \mathrm{s}$ ), which has much more significant initial values of $\omega_{x 0}$ and $\omega_{y 0}$, than in the previous example, hence has large components of $H_{x 0}$ and $H_{z 0}$, as compared with $H_{y 0}$. It results in the subdivision of the dome into two parts $\left(H_{+}\right.$and $\left.H_{-}\right)$by the inclined 2D plane, shown in white in Fig. 23a. Results of the intersection lines of the $\mathbf{e}_{2}$ ort with the dome are shown in Fig. 23a. They somehow resemble ball of wool (see Fig. 23b), especially with the knitting needles resembling the $\mathbf{H}$ and $\mathbf{e}_{2}$ vectors. However, the simulated resulting ball-of-wool lines are sitting on one hemisphere only! This hemisphere is on the side of the plane, perpendicular 
(a)

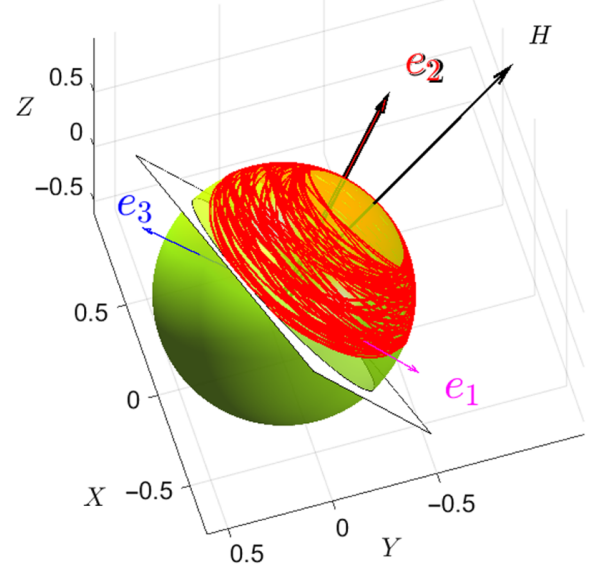

(b)

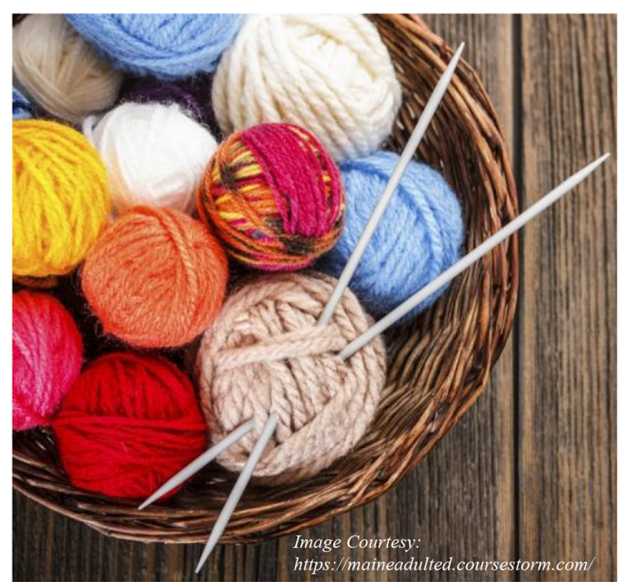

Figure 23. Ball-of-wool lines: (a) Simulation results for the case $\mathrm{I}_{\mathrm{xx}}=2, \mathrm{I}_{\mathrm{yy}}=4, \mathrm{I}_{\mathrm{zz}}=3$ (all in $\mathrm{kg} \times \mathrm{m}^{2}$ ) and initial angular velocities $\omega_{\mathrm{x} 0}=0.5, \omega_{\mathrm{y} 0}=0.5, \omega_{\mathrm{z} 0}=1$ (all in rad/s), (b) Original balls of wool, which prompted the used analogy and terminology.

to $\mathbf{H}$ vector (and we will called it $H_{+}$hemisphere). The other side of the hemisphere $\left(H_{-}\right)$ does not have any threads of the ball of wool.

This discovered new result can be used in the design of various spacecraft missions. For example, in case of the communication mission, if the spacecraft is installed in orbit with predominant rotation about an intermediate axis of inertia, and is carrying an antenna, it should be ensured that the initial direction of the angular momentum vector $\mathbf{H}$ is consistent with the source, sensed by antenna, i.e. with $H_{+}$hemisphere facing the source, otherwise spacecraft communication would be blanked for all instants of the mission. So, it matters, which side of the spacecraft, perpendicular to the axis with maximum moment of inertia, is selected: one side would be good for utilising antenna, the other side would be inoperable/terminal. The exposure efficiency of the equipment on the selected sides was explored in reference ${ }^{(22)}$.

On the same token, in some other cases, when, for example, the spacecraft is subject to directional adhere conditions (heat, radiation, flying debris) it may be advisable to reinforce

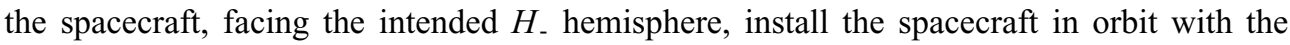
direction of the initial angular momentum pointing outwards the danger and place all sensitive equipment on the side, perpendicular to the axis with maximum moment of inertia and with positive component of the angular velocity along this direction (i.e. plus $\mathbf{e}_{2}$ in the two previously considered illustration cases).

\subsection{INERTIAL MORPHING IN ACTION: TWO-PHASE ATTITUDE DYNAMICS MANOEUVER}

Figure 24(a) shows non-dimensional angular momentum sphere with two separatrices and sets of representative polhodes for the wide range initial conditions. It also shows, as a blue bold line, a specific polhode (or hodograph of the $\overline{\mathbf{H}}$ vector) for the Phase-1 conditions: $I_{x x}=2, I_{y y}=3, I_{z z}=4, \omega_{x 0}=0.4, \omega_{y 0}=1, \omega_{z 0}=0.8$. 
(a)

$I_{x x}=2.00, I_{y y}=3.00, I_{z z}=4.00(\eta=0.500, \xi=0.500)$

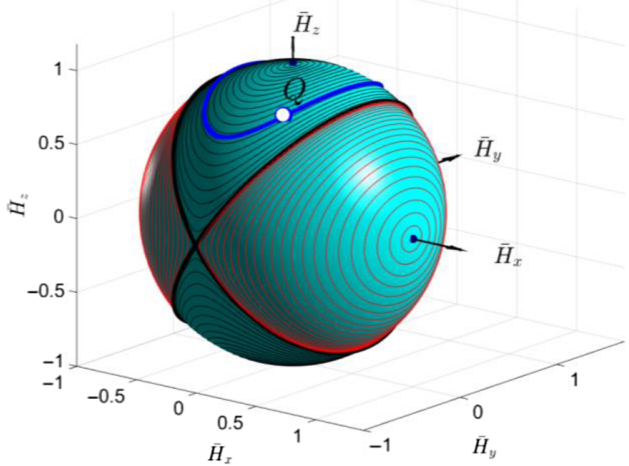

(b)

$I_{x x}=3.50, I_{y y}=3.00, I_{z z}=4.00$

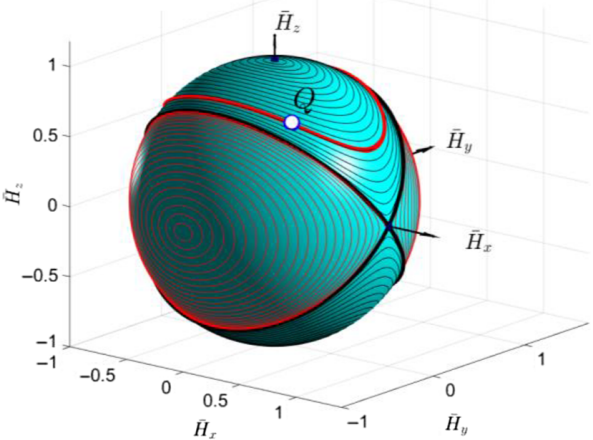

Figure 24. Non-dimensional angular momentum spheres with polhodes and separatrices and truncated specific hodographs for (a) Phase-1 (before inertial morphing) conditions: $I_{x x}=2, I_{y y}=3, I_{z z}=4, \omega_{x 0}=0.4$, $\omega_{\mathrm{y} 0}=1, \omega_{\mathrm{z} 0}=0.8$; specific hodograph shown with blue line; and (b) Phase-2 (after inertial morphing) conditions: $I_{x x}=3.5, I_{y y}=3, I_{z z}=4, \omega_{x t_{Q}}=0.7133, \omega_{y t_{Q}}=-0.7318, \omega_{z t_{Q}}=0.9016, t_{Q}=21.5 \mathrm{~s}$; hodograph shown with red line.

(a)

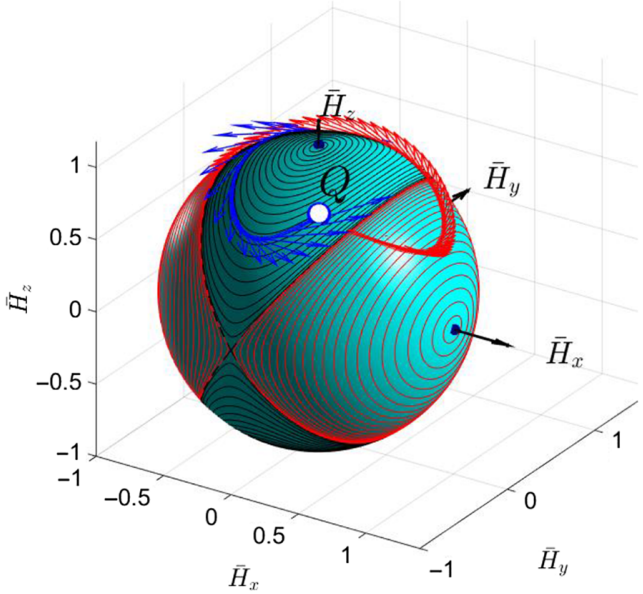

(b)

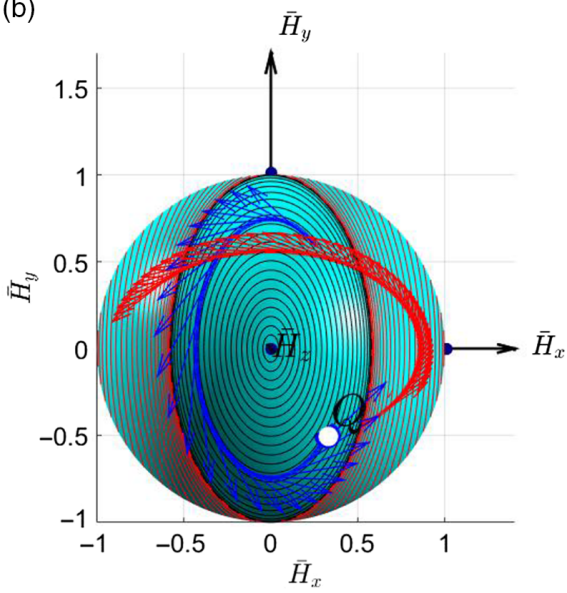

Figure 25. Illustration of the transition between Phase-1 and Phase-2 of the inertial morphing of the system: (a) side 3D view; (b) z-axis $2 \mathrm{D}$ view.

If the spacecraft possesses with inertial morphing capabilities, then the switch to any new inertial properties can be simulated and illustrated graphically. Let us assume, for illustration purposes, that the new principal moments of inertia are: $I_{x x}=3.5, I_{y y}=3, I_{z z}=4$. Then, for the Phase-2, its own non-dimensional angular momentum sphere with two separatrices and sets of representative polhodes (for the wide range initial conditions) can be also produced (see Fig. 25b). Morphing can be applied at any stage during the execution of Phase-1. For certainty, let us also assume that the morphing is rapidly applied at $t=21.5 \mathrm{~s}$ instant. Then, the new corresponding angular velocities of the spacecraft could be calculated, using equations (21). 

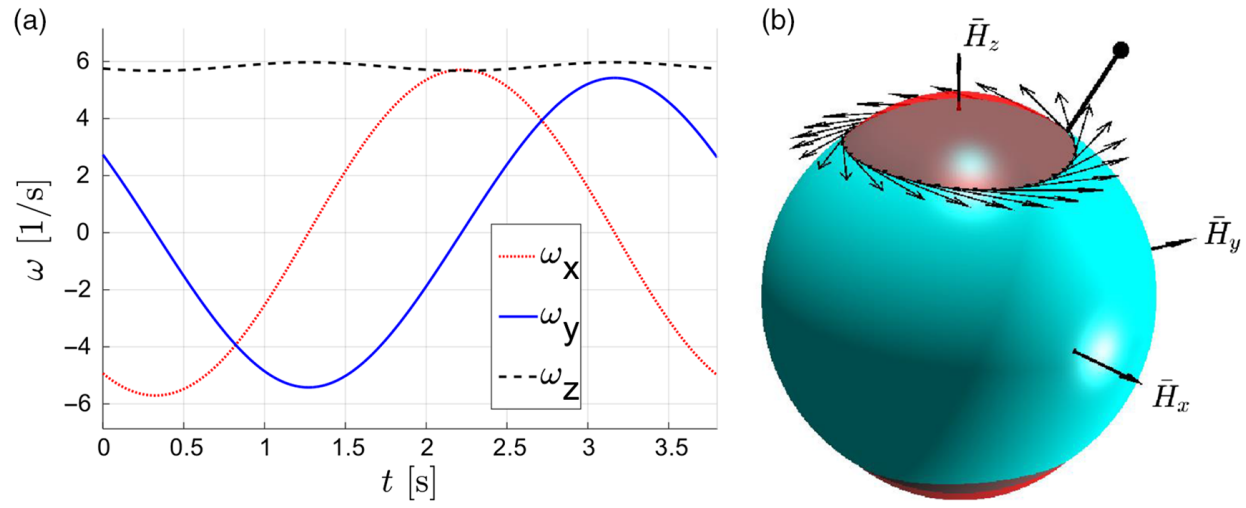

Figure 26. Illustration of the spacecraft tumbling motion: (a) time history of $\omega_{x}, \omega_{y}, \omega_{z}$ - components of its angular velocity vector $\vec{\omega}$, (b) graphical interpretation of the motion, using KEE and AMS.

\subsection{INERTIAL MORPHING IN ACTION: TRANSFER OF THE TUMBLING MOTION INTO STEADY SPIN ABOUT SELECTED BODY AXIS (THREE-STAGE STABILISATION OF THE SPACECRAFT VIA INERTIAL MORPHING AND UNSTABLE FLIPPING)}

Let assume that the spacecraft with given initial values of the moments of inertia $\left(I_{x x}=2.5, I_{y y}\right.$ $=2.4, I_{z z}=3.15$ ) is originally in arbitrary free rotation, involving all three angular velocities, as shown in Fig. 26a.

This motion can be visualised, using intersecting kinetic energy ellipsoid and angular momentum sphere, as shown in Fig. 26b. The $\overline{\mathbf{H}}$ vector of unit genuine length cannot be used for visualization, as its length is equal to one and it would not be seen at any instant, as it would be completely hided by the embracing angular momentum sphere with unit radius. Therefore, for visualisation of the instantaneous orientation of $\overline{\mathbf{H}}$ in the Fig. 26b, a black line is used with a dot at its end and extruding beyond the surface of the sphere. The hodograph of the vector is shown with a black line on the surface of the angular momentum sphere, coming strictly along the intersection between the AMS and KEE.

Let us set a task to control rotations of the system, via the changes of the values of its principal moments of inertia. In each case of using flipping mode for escaping from the closed smooth polhode, we need to apply change to moments of inertia, which could be calculated based on the parameters of the targeted separatrix, using Eqs. (7)-(8).

An example of complete set of morphings, stabilising the system, being initially in the state of tumbling, is illustrated with Fig. 27.

Figure. 27 explains the sequence and nature of the inertial changes, deliberately applied to the system. Figure 28 gives consecutive snap-shots from the simulation process, illustrating changes of the kinetic energy ellipsoid and polhodes — resultant feasible trajectories for the angular momentum vector.

It is interesting to observe, that at the initial stage of the motion of the system, its $\mathbf{e}_{2}$ body axes ort is drawing a pretty spread trajectory on the "dome" (Fig. 29a). However, after stabilization is completed, this trajectory is essentially reduced to the point (Fig. 29b). Also, at the last stage of the simulation, trajectories for $\mathbf{e}_{1}$ and $\mathbf{e}_{3}$ are very close to the equatorial plane, 

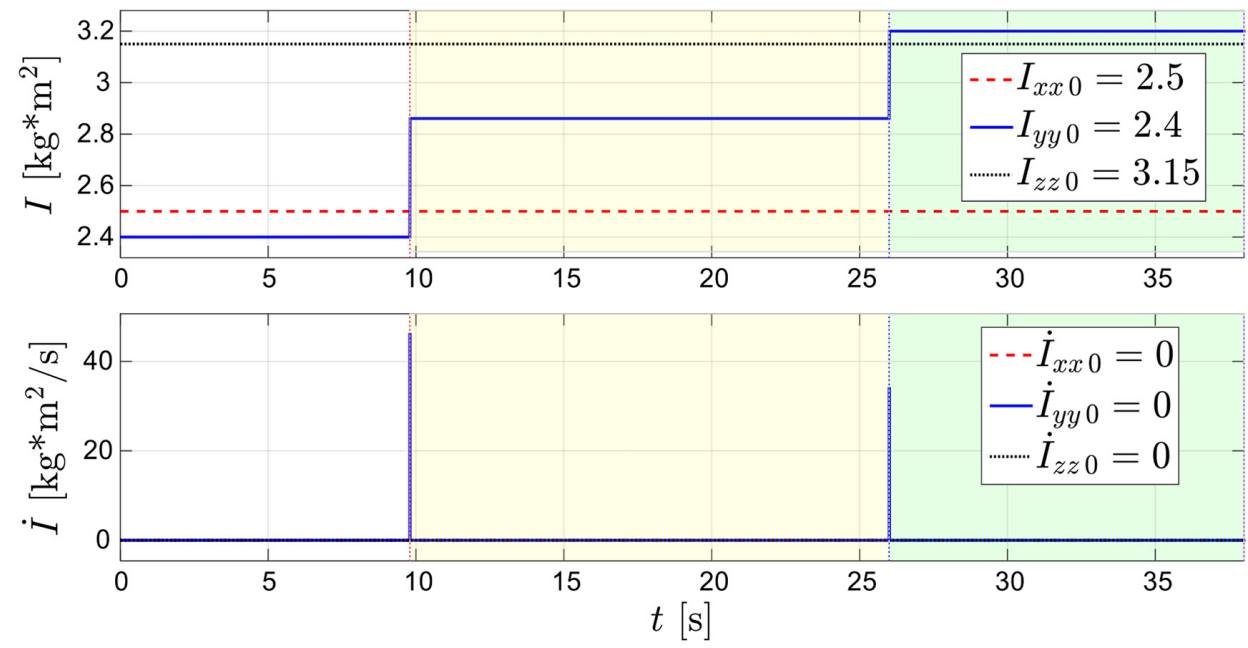

Figure 27. Three-stage stabilisation of the tumbling spacecraft via morphing: time history of the morphed principal moments of inertia $I_{x x}, I_{y y}, I_{z z}$.

(a)

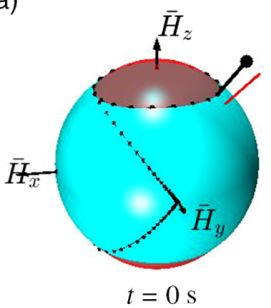

(e)

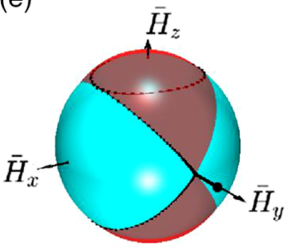

$t=15 \mathrm{~s}$ (b)

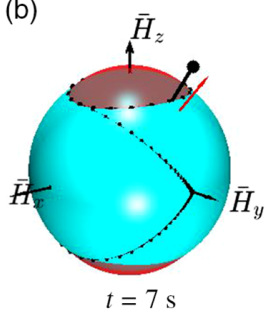

(f)

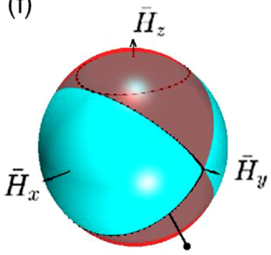

$t=19 \mathrm{~s}$

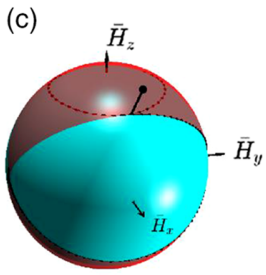

$t=9.792 \mathrm{~s}$

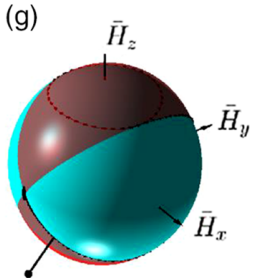

$t=22 \mathrm{~s}$

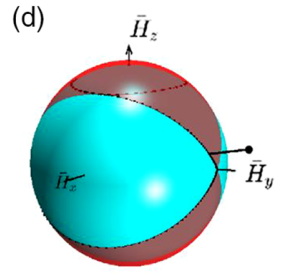

$t=12 \mathrm{~s}$

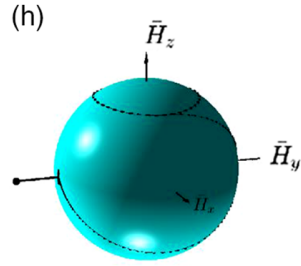

$t=26-38 \mathrm{~s}$

Figure 28. Critical instances of spacecraft stabilisation: (a) Start of the simulation; (b) Initially, hodograph is circling around $z$ axis, (c) Stage- 1 ends, transition to flipping is initiated, $t=9.792 \mathrm{~s}$; (d) approch to the saddle point-1, $t=12 \mathrm{~s}$; (e) near the saddle point-1 (possible parking or stabilisation point), $t=15 \mathrm{~s}$ (f) passing saddle point-1, $t=19 \mathrm{~s}$; (g) approach to the saddle point-2, $t=22 \mathrm{~s}$; (h) stage-2 ends and third stage starts at $t=26 \mathrm{~s}$, parking at the stable saddle point- 2 arrtactor is activated, stabilisation is completed.

which confirms that the stabilized motion is close to the rotation of the body along the direction of the angular momentum vector. The feature of the example is: the final direction of the y body axes system, selected for stabilisation in this example, is opposite to the direction of $\mathbf{H}$. If the goal of stabilisation was to have them both aligned, then third stage should be activated at instant close to $15 \mathrm{~s}$, as evidenced by the Fig. $30 \mathrm{~b}$. 
(a)

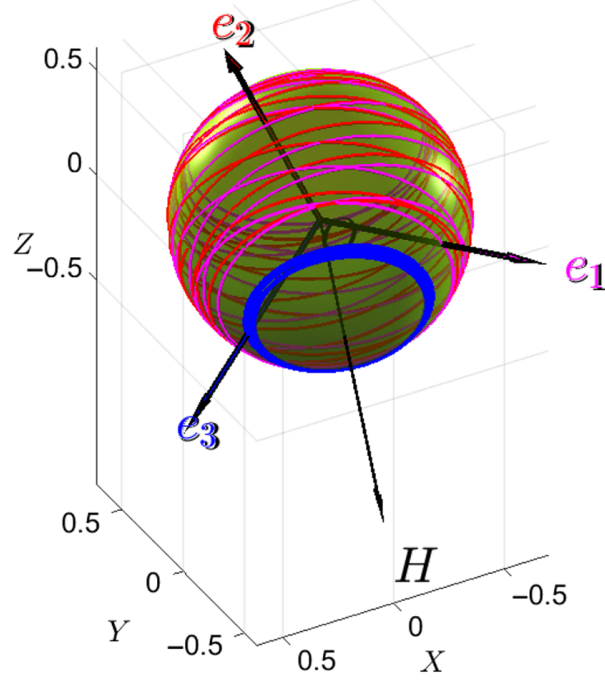

(b)

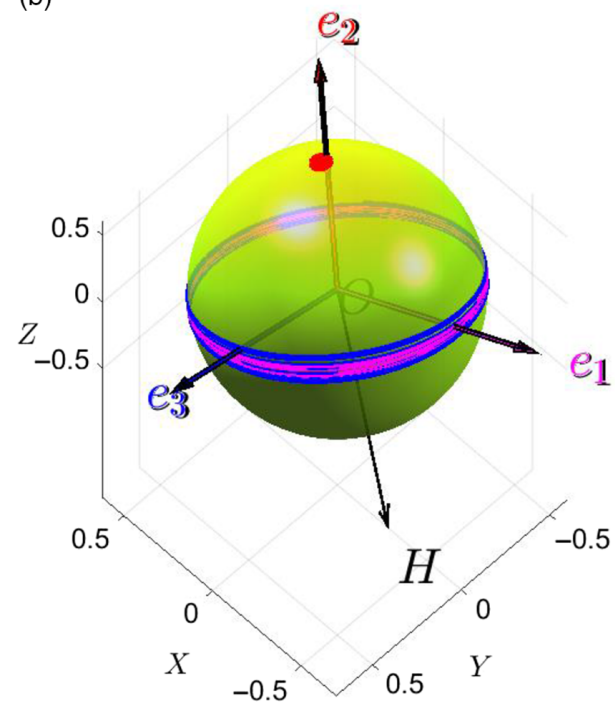

Figure 29. Balls of wool for : (a) the first stage of spacecraft motion with tumbling/coning $(t=0 \div 9.792 \mathrm{~s})$; (b) last stage of stabilisation of the spacecraft $(t=26 \div 38 \mathrm{~s})$ with $\mathbf{e}_{1}, \mathbf{e}_{2}$ and $\mathbf{e}_{3}$ intersection lines with the dome.

\subsection{COMBINED MULTI-PHASE DEMO: CONSECUTIVE PARADE OF ALL THREE ORTHOGONAL INVERSIONS, ASSOCIATED WITH $X, Y$ AND $Z$ BODY AXES}

In order to demonstrate capability of the proposed method, in Fig. 31 we present results for a single simulation case, during which the spinning body is reconfigured four times. The carefully selected scenario for the applied inertial morphing (changes in the system, leading to the change of the values of the principal moments of inertia) enables to achieve the flowing:

(1) Established flipping motion along $y$ axis (with possibility for y inversion), distinguished with a white background in Fig. 31;

(2) Established flipping motion along $z$ axis (with possibility for $z$ inversion), distinguished with green background in Fig. 31;

(3) Established flipping motion along $x$ axis (with possibility for $\mathrm{x}$ inversion) distinguished with pink background in Fig. 31.

Consequently, it has been demonstrated that the predominant spin can be consecutively passed on to any of the body axis with multiple possibilities for inversion at any stage of the stabilised motion and then stabilisation of the desirable orientation. In other words, if the object had a cube shape, based on this example, it was possible to perform transition of the spinning motion of the cube, allowing exposure of each of its six faces to the direction of the initial angular momentum vector. We call this compound demo case "all-axes inversion parade". 

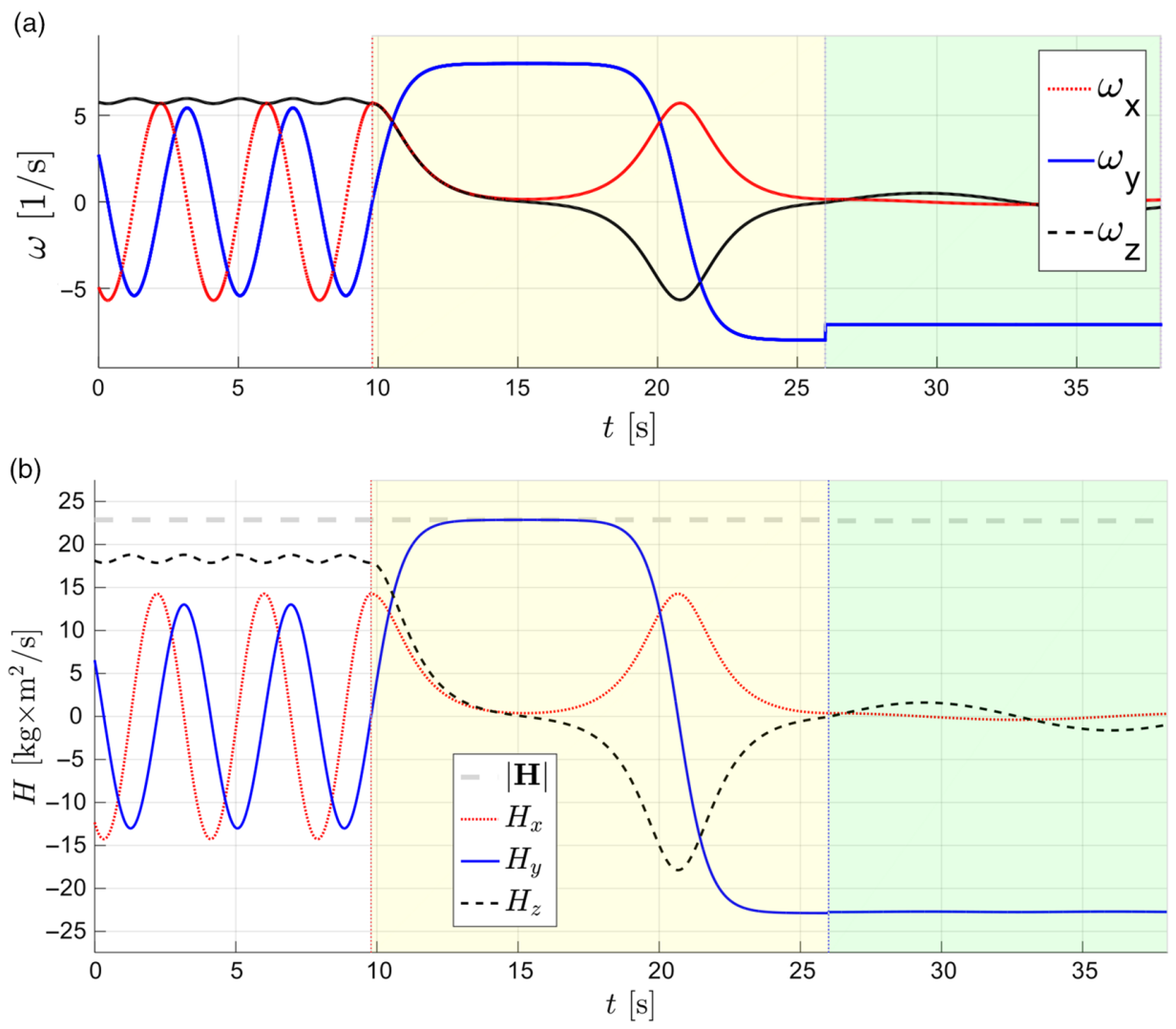

(c)

$a_{0}=[0.9349,0.9160,1.0494] ; a_{f}=[0.8839,1.0000,0.9922]$

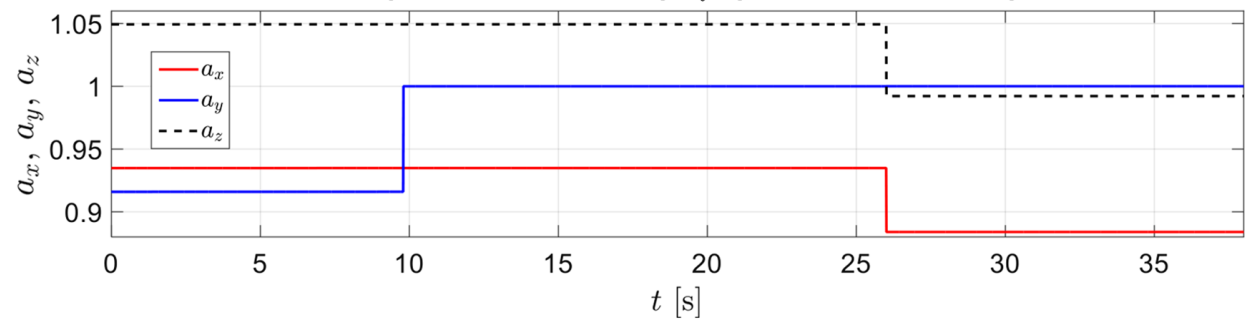

Figure 30. Time histories of the (a) $\omega_{\mathrm{x}}, \omega_{\mathrm{y}}, \omega_{z}$; (b) $H_{\text {total }}, H_{x}, H_{y}, H_{z}$ and (c) $a_{x}, a_{y}$ and $a_{z}$ during two-stage stabilisation of the tumbling spacecraft via morphing.

\subsection{ENHANCEMENT OF THE REORIENTATION AND CHANGE OF THE SPIN AXIS USING REACTION WHEELS}

For completeness of this paper, we need to mention another powerful aspect of further enhancement of the spinning spacecraft attitude control capabilities: adding one or a set of moment reaction wheels, which are often used on various space systems ${ }^{(23)}$. 
(a)
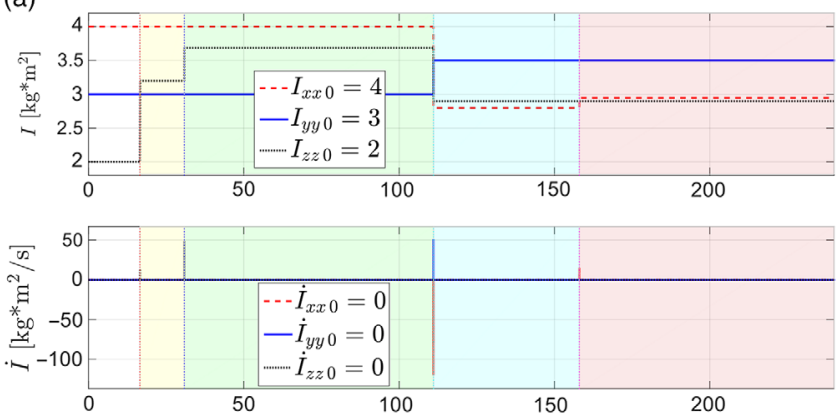

(b)

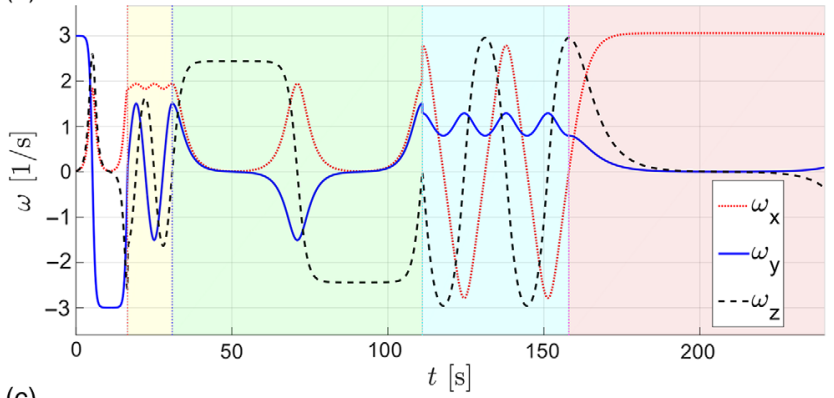

(c)

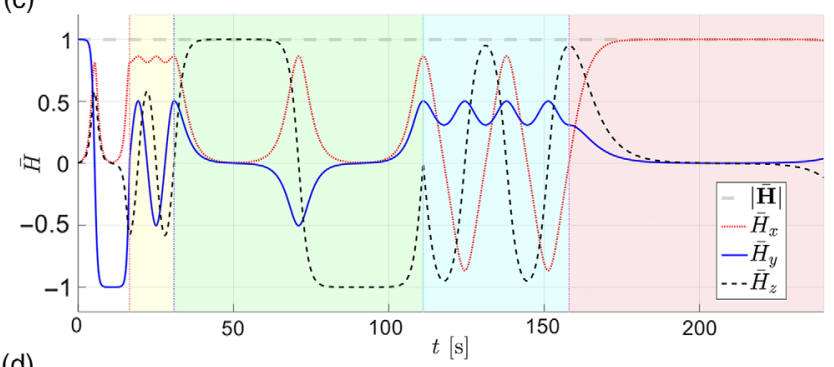

(d)

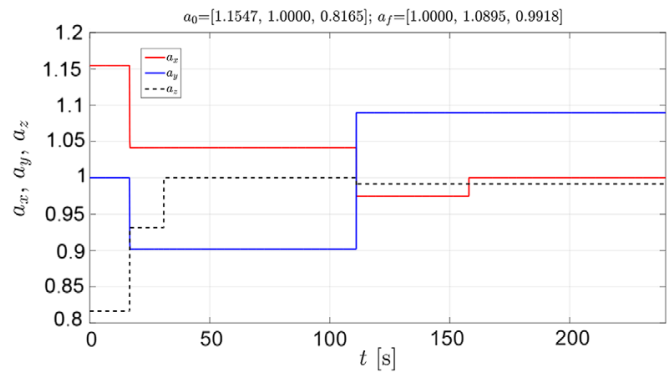

Figure 31. Time history for the following parameters during four-stage all-axes inversion parade: (a) $I_{x x}$, $I_{y y}, I_{z z} ;$ (b) $\omega_{x}, \omega_{y}, \omega_{z} ;$ (c) $H_{\text {total }}, H_{x}, H_{y}, H_{z}$; (d) $a_{x}, a_{y}$ and $a_{z}$. 


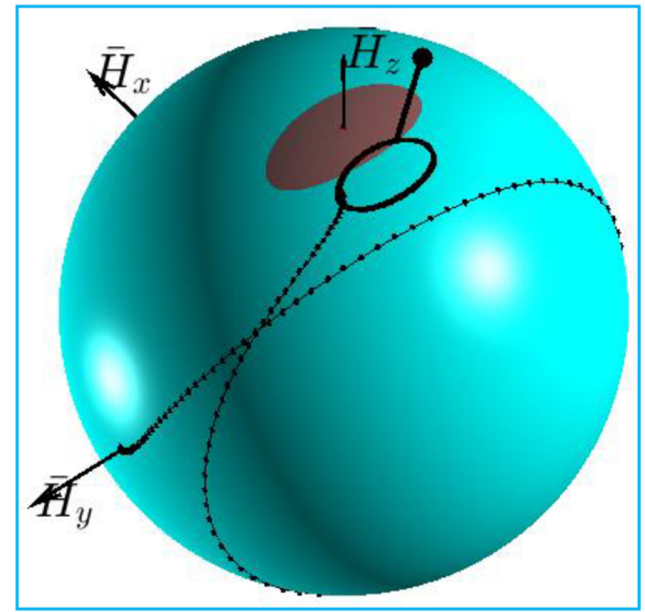

Figure 32. Shift of stabilisation point, achieved with compoung use of the inertial morphing and reaction wheel.

Differential equations of motion of the spacecraft, equipped with wheels, could be presented as follows:

$$
\begin{gathered}
{\left[\begin{array}{cccccc}
I_{x x} & 0 & 0 & 0 & 0 & 0 \\
0 & I_{y y} & 0 & 0 & 0 & 0 \\
0 & 0 & I_{z z} & 0 & 0 & 0 \\
0 & 0 & 0 & \sin \theta \sin \phi & \cos \phi & 0 \\
0 & 0 & 0 & \sin \theta \cos \phi & -\sin \phi & 0 \\
0 & 0 & 0 & \cos \theta & 0 & 1
\end{array}\right]\left\{\begin{array}{c}
\dot{\omega}_{x} \\
\dot{\omega}_{y} \\
\dot{\omega}_{z} \\
\dot{\psi} \\
\dot{\theta} \\
\dot{\phi}
\end{array}\right\}} \\
=\left\{\begin{array}{c}
\left(I_{y y}-I_{z z}\right) \omega_{y} \omega_{z}-\dot{I}_{x x} \omega_{x} \\
\left(I_{z z}-I_{x x}\right) \omega_{z} \omega_{x}-\dot{I}_{y y} \omega_{y} \\
\left(I_{x x}-I_{y y}\right) \omega_{x} \omega_{y}-\dot{I}_{z z} \omega_{z} \\
\omega_{x} \\
\omega_{y} \\
\omega_{z}
\end{array}\right\}-\left\{\begin{array}{c}
n_{\omega_{1}}+\omega_{y} l_{3}-\omega_{z} l_{2} \\
n_{\omega_{2}}+\omega_{z} l_{1}-\omega_{x} l_{3} \\
n_{\omega_{3}}+\omega_{x} l_{2}-\omega_{y} l_{1} \\
0 \\
0 \\
0
\end{array}\right\}
\end{gathered}
$$

Even simple preliminary cases, involving one wheel and not sophisticated wheel's controls, enabled us to find significant influence of this enhancement on performance of the system. In particular, it was possible to significantly influence the period of inversion, make inversions asymmetrical (see Fig. 32), etc. The authors intend to explore these capabilities in more detail in the future works.

\subsection{CONCLUSIONS}

In this paper, a methodological framework for enhancing attitude control of the spacecraft, using inertial morphing, is presented. It is based on the geometrical interpretation of equations of non-linear motion (involving non-dimensionalised angular momentum unit spheres and kinetic energy ellipsoids) and features amazing simplicity, while giving impressive 
advanced range of tools for preliminary designs of the specific missions. A comprehensive non-dimensional mathematical construction/formalisation to formulate and solve wide range of attitude dynamics and control problems is presented. Applications of this methodology are vast, including (but not limited to) the following possible applications:

(a) Inertial morphing that permits stopping (i.e. completely switching off) of the unstable flipping motion of the spinning or tumbling spacecraft, if these motions are undesirable, by translating the motion into the regular spin. Similarly, this methodology enables initiation (i.e. switching on) of the spinning spacecraft unstable periodic flipping. The combination of switching on and switching off capabilities, without using traditional gyroscopic devices, can be used for the inversion of the spacecraft, where the forward/backward flying spacecraft could be easily converted into the backward/forward flying system. Furthermore, this technique can be used for boosting (accelerating) or decelerating spacecraft by only one thruster (i.e. for thruster direction control). It should be stressed that two classes of possible solutions for switching off the flipping motion were found, which presents multiple alternatives during missions planning/design.

(b) Inertial morphing can be very effective for controlling/changing the frequency of the flipping motion within a very wide range. However, it was shown that there is a minimum (i.e. low bounding limit) for the period of these oscillations.

(c) Using inertial morphing, a method of reduction of the compound rotation of the spacecraft into a single stable predominant rotation around one of the body axes was proposed. This is achieved via multi-stage morphing. One of the transformation stages employs transition of the system into unstable, flipping motion, enabling the transfer of the motion into a special type, which could be represented with a polhode, situated close to the separatrix. After this instalment into the separatrix, the final stage of the transition is typically dedicated to conversion unstable motion into the stable. With the capability of this transfer of the spacecraft spin into a single axis spin, aligned with the angular momentum direction, spacecraft essentially could perform three types of inversions, associated with any of three body axis. In order to demonstrate capabilities of the method, an all-axes inversion parade was presented, during which the spinning system was transitioned through three consecutive stages with inversion, associated with each of the body axes, $x, y$ and $z$. This is in contrast with the classical Dzhanibekov's Effect demonstration, where only one axis inversion is possible.

(d) Attitude orientation of different sides of the spacecraft during various spinning/tumbling scenarios was investigated and a simple ball of wool method to determine the most advantageous sides of the spacecraft for attaching special equipment, like antennae and/or solar batteries, was proposed. It has been discovered, that for the motion, resembling Dzhanibekov's Effect flipping, one side of the prism-shaped spacecraft, perpendicular to the major axis of inertia (named as $H_{+}$), would be always sensed from the specified direction, whereas the second side (named as $H_{-}$), would never be sensed from the same specified direction. This important finding suggests the strategies for proper placement of the sensitive equipment on the right sides of the spacecraft and for reinforcement of the side, which could be deliberately made exposed to the adverse directional conditions (heat, radiation, flying space debris, asteroid belts, etc.)

This paper presents examples of the implementations of the inertial morphing. These methods include, but not limited to: (i) use of mechanical actuating systems and/or pre-compressed springs to re-position masses within the spacecraft; (ii) use of heavy liquids and/or magnetic 
liquids and associated mechanisms and valves to change the mass distribution within the system, employing passive inertial forces and/or controlled magnetic field forces to move these liquid medias; (iii) use mass ejection, evaporation, ablation, solidification to change the system mass, etc.

It has been also demonstrated that a reaction wheel system could further enhance spacecraft capabilities, enabling changes into the angular momentum of the system offering full access to the inertial morphing strategies.

\section{REFERENCES}

1. Trivailo, P.M. and KoJIma, H. Enhancement of the spacecraft attitude dynamics capabilities via combination of the inertial morphing and reaction wheels (keynote paper and presentation). [Peer Reviewed], Transactions of the 18th Australian International Aerospace Congress (AIAC2019), incorporating 27th International Symposium on Space Flight Dynamics (ISSFD). Engineers Australia, Royal Aeronautical Society. Date of Publication: 24/02/2019. ISBN: 978-1-925627-21-3. pp. 1093-1122.

2. Trivailo, P.M. and KoJima, H. Discovering method of control of the "Dzhanibekov's Effect" and proposing its applications for the possible future space missions. - Transaction of JSASS (The Japan Society for Aeronautical and Space Sciences), Aerospace Technology Japan, 2019, 17, (1), 72-81. doi:10.2322/tastj.17.72.

3. Trivailo, P.M. and KoJima, H. "Augmented control of inversion of the spinning spacecraft, using inertial morphing", - Paper IAC-18,C2,3,5,x45333, Proceedings of the 69th International Astronautical Congress (IAC), Bremen, Germany, 01-05 October 2018. pp. 16.

4. Trivailo, P.M. and KoJIma, H., "Re-Discovering 'Dzhanibekov's Effect' Using Non-Linear Dynamics and Virtual Reality", - Paper IAC-17,E1,7,10,x41083, Proceedings of the 68th International Astronautical Congress (IAC), Adelaide, Australia, 25-29 September 2017. -IAC, 17, pp. 11394-11407, - 14 pp.

5. Trivailo, P.M. and Koлmma, H., "Utilisation of the 'Dzhanibekov's Effect' for the Possible Future Space Missions", - Paper ISTS-2017-d-047/ISSFD-2017-047, Proceedings of the Joint Conference: 31st ISTS, 26th ISSFD \& 8th NSAT, Matsuyama, Japan, 3-9 June 2017. pp. 10.

6. Murakami, H., Rios, O. and Impelluso, T.J., "A theoretical and numerical study of the Dzhanibekov and tennis racket phenomena," Journal Applied Mechanics, 83, (Sept. 08, 2016), (11), 111006 (10 pages). Paper No: JAM-16-1017, doi:10.1115/1.4034318.

7. WIE, B., "Space Vehicle Dynamics and Control", Second Edition, AIAA Education Series, 2008, pp. 966, doi:10.2514/4.860119.

8. Moler, C., "Tumbling Box ODE. (Cleve's Corner: Cleve Moler on Mathematics and Computing)," Posted by Cleve Moler, August 10, 2015. http://blogs.mathworks.com/cleve/ 2015/08/10/tumbling-box-ode/ (accessed Aug 18, 2019).

9. Beachley, N.N., "Inversion of spin-stabilized spacecraft by mass translation - some practical aspects," Journal of Spacecraft, 8 (1971), pp. 1078-1080.

10. Theory of the Reversing Earth, http://www.scientiapress.com/theory-of-the-reversing-earth (accessed Aug 18, 2019).

11. Shkaplerov, A. and Burbank, D. Experiments on board of the International Space Station (Expedition \#30, 2011). https://youtu.be/LzVItPwiQyI (published February 11, 2013; accessed Aug 18, 2019).

12. WaKATA, K. Mysteries of Rotational Movement (Onboard of the ISS, Expedition \#38, 2014), JAXA. https://youtu.be/QhSN2eua14I?t=309 (published Nov 25, 2013; accessed Aug 18, 2019).

13. FoRD, K. Working with the Spheres Satellites Onboard of the ISS (Expedition \#34, 2013) https://youtu.be/dsXOxcDSBLQ?t=108 (published Mar 13, 2013; accessed April 09, 2017).

14. Kawano, D.T., "A Tumbling T-Handle in Space," Rose-Hulman Institute of Technology. http://rotations.berkeley.edu/?page_id=2472 (accessed Aug 18, 2019).

15. Landau, L.D. and Lifshitz, E.M., Mechanics. Vol. 1 (In Russian), 4th ed. Moscow, "Nauka”. 1988, pp. 216.

16. CurTis, H. Orbital Mechanics for Engineering Students, Elsevier, 2005, pp. 675. 
17. YAO, Q. and GE, X., Optimal control of stretching process of flexible solar arrays on spacecraft based on a hybrid optimization strategy, Theoretical \& Applied Mechanics Letters, 7, 2017, pp. 258-263.

18. NOACK, D. In-orbit verification of a fluid-dynamic attitude control system. Paper 2019-d-041, Proc. of the 32nd ISTS \& 9th NSAT (The International Symposium on Space Technology and Science and Nano-Satellite Symposium), Fukui, Japan, 15-21 June 2019.

19. Hwang, J.T., Lee, D.Y., Cutler, J.W. and Martins, J.R.R.A., Large-scale multidisciplinary optimization of a small satellite's design and operation. Journal of Spacecraft and Rockets, 51, (5), September-October 2014, pp. 1648-1663. DOI:10.2514/1.A32751.

20. Ohashi, K., Chujo, T. and Kawaguchi, J., motion planning in attitude maneuver using nonholonomic turns for a transformable spacecraft. - The 28th JAXA Workshop on Astrodynamics and Flight Mechanics, Sagamihara (Japan), 2018, pp. 6.

21. Hernando-Ayuso, J., Baresi, N. and Chujo, T., Orbit design and insertion for the jaxa transformable spacecraft, The 28th JAXA Workshop on Astrodynamics and Flight Mechanics, Sagamihara (Japan), Paper B-26, 2018, pp. 8.

22. Dorrington, G. and Trivailo, P.M. Utilising the 'Chaotic' Tumbling of CubeSats. [Peer Reviewed], Transactions of the 18th Australian International Aerospace Congress (AIAC2019), incorporating 27th International Symposium on Space Flight Dynamics (ISSFD). Engineers Australia, Royal Aeronautical Society. Date of Publication. 24/02/2019. ISBN: 978-1-92562721-3, pp. 1390-1412.

23. Terui, F., Kimura, S., Nagai, Y., Yamamoto, H., Yoshihara, K., Yamamoto, T. and Nakasuka, S., Moon tracking attitude control experiment of a bias momentum micro satellite " $\mu$-LabSat", Transactions of the Japan Society for Aeronautical and Space Sciences, 48, (159), 2005, pp. 28-33. 